\title{
Internet Governance as Forestry: \\ Deriving Policy Principles from Managed Complex Adaptive Systems
}

TPRC 2008

\begin{abstract}
This paper proposes a principles-based approach for meeting regulatory goals that suits the dynamics of the internet/web.

Innovation in technology, business and culture constantly changes the context in which regulators have to make communications policy. However, there are abiding policy imperatives like public safety, consumer protection, and economic vitality which do not change. Policy makers need an approach which reconciles these competing pressures.

The internet/web can be understood as a managed complex adaptive system, such as a forest or other ecosystem. A review of systems theory and the experience of ecosystem management yields four "ecosystem management principles": flexibility - determining ends, not prescribing means; delegation - giving markets and civil society the first shot at meeting social needs; big picture taking a broad view of the problem and solution space; and diversity - enabling multiple solutions to policy problems by encouraging competition and market entry.

The metaphor of internet/web governance as forestry, or managing any complex adaptive system, can therefore guide regulatory intuition in communications. The utility of such an approach is illustrated in four policy areas: video services; telecom fees and taxes; service accessibility for the disabled; and access to emergency services.
\end{abstract}

Pierre de Vries

Research Fellow, Economic Policy Research Center (EPRC)

University of Washington, Seattle

pierredv@hotmail.com

/d08 /080812 
I. Introduction 3

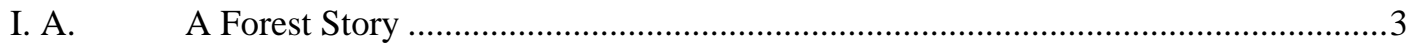

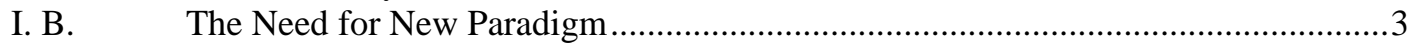

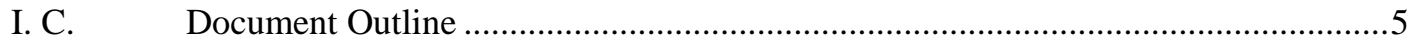

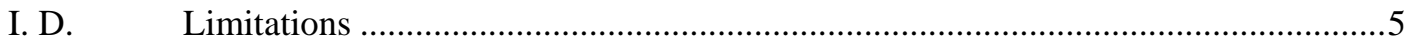

II. Permanent Policy Imperatives 6

III. The Internet/Web: Characteristics, Paradigms and Metaphors 8

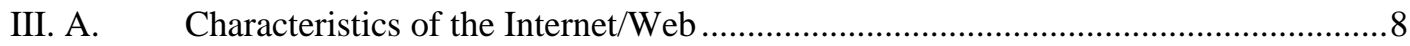

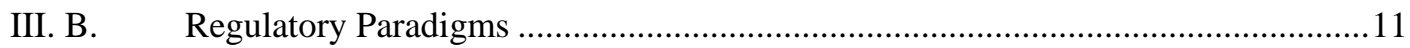

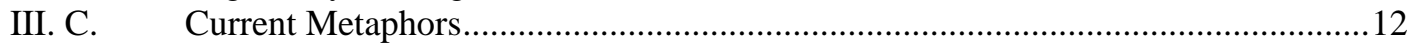

IV. The Forestry Metaphor 14

V. Insights from Managing Complex Adaptive Systems 16

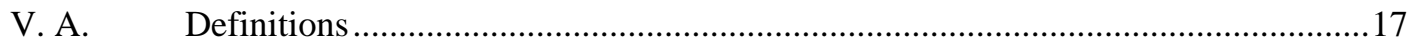

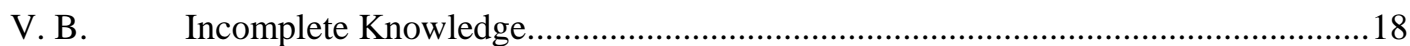

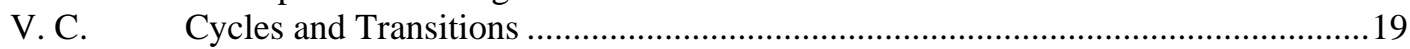

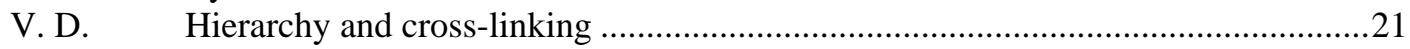

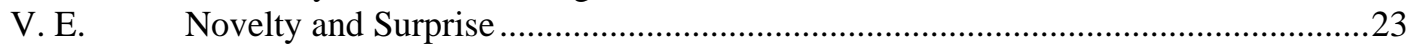

VI. The Four Principles 26

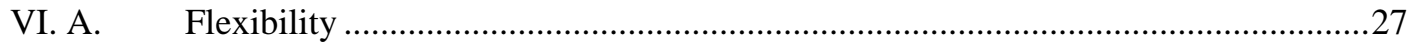

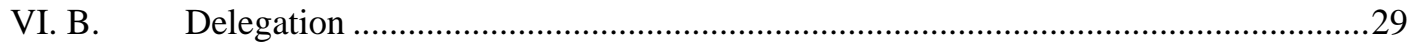

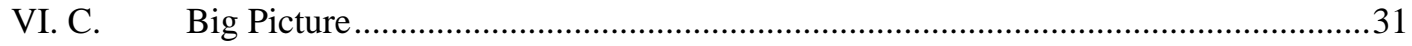

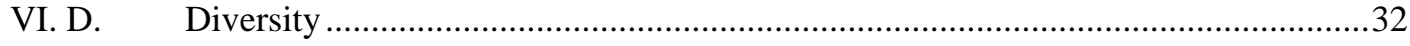

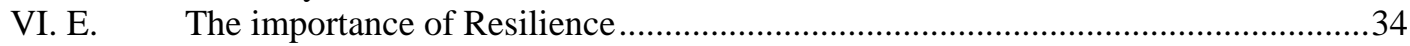

VII. The Principles in Action 35

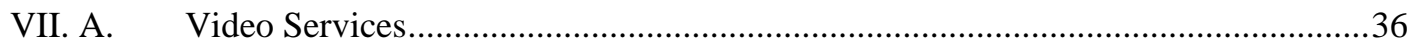

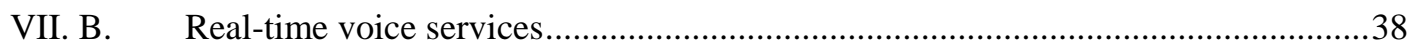

VIII. Refinements and Generalizations 41

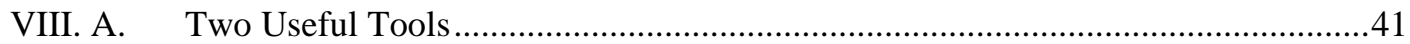

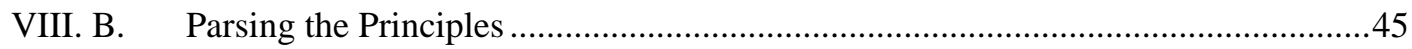

VIII. C. Using the Principles outside Government …….........................................................47

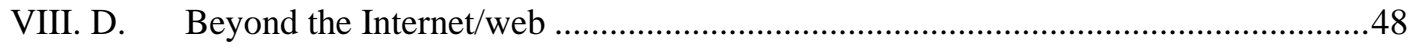

IX. Open Questions 49

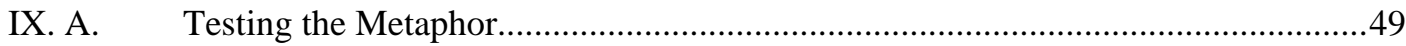

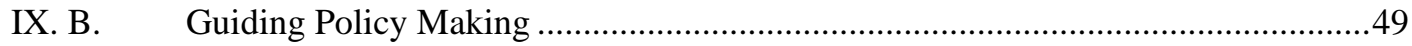

IX. C. Applying System Theory to the Internet/Web...........................................................50

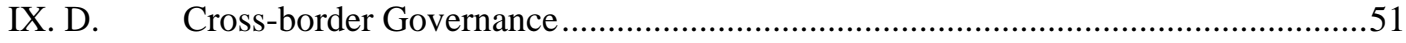

X. Conclusions 52

XI. Acknowledgments52

XII. References 53 


\section{INTRODUCTION}

\section{A. A Forest Story}

On Monday, July 10, 1989 a tornado blew down Cathedral Pines in Cornwall, Connecticut. ${ }^{1}$ It had been a magical place: an old-growth forest of white pine trees untouched since 1800, where lovers courted, couples got married, and families came for Sunday outings. After the storm had passed it was a tangled mess of lumber.

Arguments over its future started quickly. Some worried about the fire risk of the piles of lumber and brush. Many wanted a concerted effort to recreate the pine forest as it had been. The Nature Conservancy, its owners, believed that nature should be left to take its course.

Unfortunately there is no single course to perfection for nature to take. The notion of "forest succession", the eternal march of untouched wilderness towards a stable "climax community" of towering old growth, is long discredited. ${ }^{2}$ Left to its own devices, Cathedral Pines could re-grow in many possible ways. While it probably had an underlying inclination towards pine-forest succession, chance could push it in very different directions: a severe fire could delay forest recovery for decades, or a mild one could kill oak saplings and let pine seedlings flourish; a soaring deer population might wipe out the pines and make an opening for spruce; or an exotic non-native plant could take over.

Humans, in fact, created Cathedral Pines: the site was logged by the first generation of white settlers late in the $18^{\text {th }}$ century, and loggers removed hardwood trees in 1840 , leaving the pines to grow unchecked. Before them, Native Americans probably regularly burned the area to provide mixed grassland for hunting and foraging. Reconstructing it to some earlier state would require a decision about which prior condition was the "right" one.

Some human interests were eventually taken into account, but only insofar as fire risk was concerned. The Nature Conservancy decided, after negotiations with adjacent property owners and the selectmen of Cornwall, to leave the fallen trees untouched, except for a fifty-foot clear-cut firebreak around the perimeter.

The story of Cathedral Pines has many morals. Nature doesn't have a goal in mind: the contingencies of the world are endless, and consequential. Nature and culture cannot be separated: human management is unavoidable and necessary. And not least: any management process is sure to be contentious, and any result will displease some people.

The internet/web is rather like a forest. For some it is a resource to be exploited, while others see it in idealistic and even romantic terms. It is constantly changing, and there is no pre-determined optimal outcome. Some management is inevitable - but always contentious.

\section{B. The Need for New Paradigm}

Modern communication systems are enormously intricate and dynamic, and everyone has a stake in their successful operation. Policy makers are held to account by their constituencies for success and failure, and thus communications policy is unavoidable. However, the complexity of the topic

\footnotetext{
${ }^{1}$ This story is taken from Pollan (1991) Ch. 10. For images of the remaining intact portion of the stand, see http://terrasacra.com/cathedral_pines.asp

2 Budiansky, Stephen “Chaos in Eden” New Scientist 14 Oct. 1995. Available (subscription may be required): http://www.newscientist.com/article/mg14819993.900-chaos-in-eden.html
} 
means that the basis for taking regulatory action, let alone the precise policies to be deployed, is a very knotty problem.

This paper lays out an approach to deciding whether and how government actors should regulate the internet/web. ${ }^{3}$ It provides a framework for action that reflects the underlying system dynamics, balances conflicting interests, and maximizes social benefits. The approach was developed and is applied to the internet/web, but it applies more generally to any policy problem that involves an intricate and evolving interplay of social, technical and commercial forces.

This approach proposes to bridge from day-to-day contingencies to policy plans and rules by using principles to guide policy making. While I will discuss some test cases, I do not prescribe rules; it will ultimately be up to policy makers themselves to apply the principles in their specific context.

One should not expect this approach, or any other, to give unique, unambiguous, or uncontested answers to complex policy problems. The consequences of a principle may be arguable, there may be debate about the applicability of competing principles, or the principles may imply conflicting courses of action. Policy making is judgment, not arithmetic. Questions will ultimately be settled by reasoned argument as in a court of law, rather than by analytical calculation.

Internet technologies and web entrepreneurs are constantly reconfiguring social interactions, industry structures, and commercial practices. New ways of doing things often don't fit well into old regulatory structures. Perennial policy questions about the public interest, economic growth and social impacts need to be revisited because the technical drivers and social dynamics have become significantly more complex. For example, traditional methods of controlling or promoting content that presumed a limited number of producers and distribution channels do not work in the face of millions of user-generated videos and cross-border media flows; mandates for making services accessible to the disabled are complicated, and sometimes rendered obsolete, by the myriad ways content is presented; and traditional ways of raising revenue via fees on telephone calls become questionable if traffic shifts to a wide variety internet-enabled voice services.

The novelty and complexity of the internet/web requires new mental models. The traditional “silo” metaphor doesn't work any more, and the techno-centric “layers” model doesn't reflect social and regulatory dynamics. Current metaphors for the internet, from pipes and highways to tools and places, don’t fully capture its complexity, emergent behavior, or its political dimension.

It is helpful to imagine the internet as a large ecosystem, and communications policy as ecosystem management. These are examples of managed complex adaptive systems, and I will turn to this literature for lessons and inspiration. From complex system characteristics like incomplete knowledge, nesting, cycles, perpetual novelty, and the importance of resilience I will derive the four Ecosystem Management Principles for guiding internet/web policy:

1. Flexibility. Determine ends, not means. Describe and justify the outcomes sought, not the methods to be used to achieve them.

2. Delegation. Most problems should be solved by the market and society, not by government. Government's role is to provide proper incentives and guidance, and to intervene to solve critical shortcomings.

3. Big Picture. Take a broad view of the problem and solution space. Prefer generic to sector-, technology-, or industry-specific legislation.

\footnotetext{
${ }^{3}$ I use the term "internet/web" to highlight that one needs to consider both engineering-focused data transport (one of the main connotations of "the internet") and human-centered activities ("the web") in order to fully understand today’s communication landscape.
} 
4. Diversity. Allow and support multiple solutions to policy problems. Encourage competition and market entry.

These principles apply to any managed complex adaptive system, including any industry that is being transformed by the forces that have driven the internet/web; this paper, however, focuses on the web.

The resulting approach leans towards laissez faire, but has a clear role for government. The model is not that the internet/web is a pristine wilderness, untouched by human hands and "red in tooth and claw"; rather, it is a managed ecosystem where societal needs and human agency contend with the self-organizing complexity of the biological system. As Pollan (1991) describes a garden, it is "a place, both real and metaphorical, where nature and culture can be wedded in a way that can benefit both.”

\section{C. Document Outline}

After this introduction, Section II, Permanent Policy Imperatives, discusses the five (plus one) permanent public interest mandates that policy makers must satisfy, regardless of technology or industry structure.

Section III, The Internet/Web: Characteristics, Paradigms and Metaphors, is a survey of the attributes of the internet/web that shape the problems of contemporary policy making, and a review current regulatory paradigms and guiding metaphors. It can be skipped with little loss by anyone familiar with the field.

After introducing this paper's guiding analogy in Section IV, The Forestry Metaphor, the recurring themes of the underlying theory are reviewed in Section V, Insights from Managing Complex Adaptive Systems.

The four guiding principles are described in detail in Section VI, The Four Principles. After an illustration of their use in Section VII, The Principles in Action, I return to an analysis of the policy framework and its implications in Section VIII, Refinements and Generalizations. Opportunities for future work are covered in Section IX, Open Questions.

Section X concludes, and is followed by acknowledgements and references.

Short cuts:

- $\quad$ Section V, Insights from Managing Complex Adaptive Systems (pp. 16-26), can be omitted by those familiar with the complex systems and ecosystem management literature.

- A reader with limited time who is interested in the policy framework should skip ahead to Section VI, The Four Principles (p. 26), and Section VIII, Refinements and Generalizations (p. 41).

- Someone who wants a flavor of the implications of this approach without immersing themselves in theory should focus on Section VII, The Principles in Action (p. 35).

\section{D. Limitations}

This paper is one step towards constructing a principles-based regulatory framework based on lessons learned from managed complex adaptive systems. Much remains to be done. I summarize here the research topics discussed in Section IX, Open Questions.

To prove its worth, the approach needs to be applied in detail to specific policy question; this paper illustrates its application in a number of areas, but at a survey level. 
The policy recommendations lean towards laissez faire, though with a clear role for government. It is not clear to what extent this is a consequence of adopting the complex systems metaphor, or the result of my biases.

This paper proposes ways to deal with policy problems, which it takes as given. Can complex systems thinking also help policy makers to diagnose and characterize problems before attempting a solution?

If the internet/web is analogous to managed ecosystems, then many of the techniques developed in that field should be applicable. One should be able to simplify the complexity of the internet/web by identifying the key processes, and a limited number of variables that control them, at a variety of scales in a hierarchical structure. One should also be able to develop metrics to track the overall (emergent) performance of the internet/web.

The experience of cross-border ecosystem management could provide insights into international governance issues on the internet/web. This has not been addressed in any detail here.

\section{PERMANENT POLICY IMPERATIVES}

Societies have many social and economic interests, and regulation is one of the tools for achieving them. The underlying goal is to increase the welfare of citizens. The responsibility of policy makers to ensure the delivery of public interest benefits does not change amid the flux of technological and commercial innovation. However, a substantial socio-technical reconfiguration, such as that wrought by the internet/web, necessitates revisiting the regulatory tactics used to pursue the goals.

The broad social objectives are providing public goods ${ }^{4}$, and generating resources. Policy makers need to ensure that services necessary to public welfare are provided where economic incentives alone don't suffice; three key themes are public safety, consumer protection, and protecting cultural values, particularly regarding speech and content. They are also responsible for two kinds of resource management: generating revenue for government programs, which is a goal in its own right even though it supports all the other activities; and achieving national economic objectives, including ensuring open and competitive markets, and managing scarce public resources. ${ }^{5}$

Public Safety. Protecting citizens is a primary responsibility of government. Policy concerns regarding the internet/web include access to emergency services like police and fire fighting, law enforcement surveillance, data retention, and child safety.

Consumer Protection. Policy makers take action when lawmakers conclude that commercial activity needs to be circumscribed in the public interest. Topics in communications policy include privacy, fraud, fair billing/pricing, disclosure of terms of service, access for those with disabilities, device certification, universal service, and digital inclusion.

Culture and Values. In order to protect and express a culture's values, policy makers seek to limit some kinds of speech and promote others. The internet/web touches many issues in this arena, including censorship (eg, of subversion, hate speech, obscenity, violence), advertising rules, protecting cultural diversity, and promoting local content.

\footnotetext{
${ }^{4}$ A public good is one that, once provided to one user, must be provided in the same amount to all users.

${ }^{5}$ The EU Framework Directive states its objectives in similar but not identical terms: to encourage competition in electronic communications markets; to improve the functioning of the internal European market; and to protect basic user interests that would not be guaranteed by market forces. See eg http://icttoolkit.infodev.org/en/PracticeNote.514.html
} 
Government Revenue. Money needs to be raised and redistributed by federal, state and local treasuries; this includes taxes, fees, levies, subsidies, and tax breaks. Issues under this heading in telecommunications include universal service levies, registration fees for communications services, and spectrum auction revenues.

Economic Vitality. A healthy market produces goods and services that citizens value. Policy topics include fostering innovation, protecting property rights, setting up and regulating markets, facilitating market entry, and addressing market failures; ${ }^{6}$ encouraging competition, interoperability and interconnection; managing public goods; and growing domestic capacity through industrial and trade policy. Internet/web policy has a particular bearing on allocating spectrum, technology standards, market power of infrastructure providers, interoperability, broadband build-out, and network management.

There is, in fact, a "sixth imperative" that is personal rather than social: the regulator's pursuit of self-interest. ${ }^{7}$ Policy makers are not, in the end, guided by altruistic motives. They are expected to pursue the public interest, but their own resources are not at risk; these are provided by tax payers and those affected by regulation. Conversely, individual voters have so little at stake that collectively they are apathetic about the political process. Lobbies that have a well-defined interest have a disproportionate influence. Individual bureaucrats will tend to make decisions on the basis of career advancement interests; techniques include policies developed to protect the regulator's job or deflect criticism when something goes wrong, or measures intended to favor potential future employers. Individual policy makers also seek funds for their own personal or political use through campaign contributions, earmarks, kickbacks, bribes, etc. Offering public services through multiple agencies and other techniques of delegation may off-set these effects to some extent.

These goals can be subsumed under a broader rubric: productivity and stability. Productivity broadly construed includes material welfare as well as cultural benefits and social justice. Since innovation is a proven source of social welfare, policy makers need to create an environment where beneficial serendipity occurs. ${ }^{8}$ The policy maker must also ensure that productivity continues uninterrupted; this requires the system to be relatively stable. However, given the susceptibility of complex systems to cycles and shocks, resilience is the best one can hope for. The four principles that will be introduced below can roughly divided into two that mainly foster innovation (flexibility and delegation) and two that build resilience into the system (big picture and diversity).

The policy imperatives sometimes point in different directions; for example, economic vitality might indicate a market solution, while the need to protect culture and values might motivate intervention. The self-interest imperative often works at cross purposes with the others, since a policy maker's personal interests may conflict with society's needs. When imperatives are at odds, regulators should identify which one is decisive for a particular case, and overweight it relative to the others. In other words: one cannot a priori put the imperatives in an ordinal list.

Delivering on the policy imperatives is the responsibility of government, but regulation is not the only means of doing so. There is often a choice between using the market to generate innovation, or regulation to mandate behavior. (They are not mutually exclusive; markets require government to create stable frameworks, and governments depend on markets to generate wealth.) Given the slow

\footnotetext{
${ }^{6}$ Amendola \& Pupillo (2007) argue that regulatory intervention should be limited to “enduring bottlenecks”. Since the internet/web system is not in equilibrium, like most if not all markets, it is hard to predict whether a bottleneck will endure. The best one can probably do ex ante is to look out for entrenchment.

${ }^{7}$ This topic is often studied under the rubric of public choice theory. See eg http://en.wikipedia.org/wiki/Public_choice, http://www2.chass.ncsu.edu/garson/pa765/publicchoice.htm, http://www.econlib.org/library/Enc/PublicChoiceTheory.html

${ }^{8}$ Of course, it is not the only source of welfare, and is a source not only of welfare.
} 
pace and unintended consequences of regulation, delegation to markets and civil society can often achieve goals more reliably than intervention. For example, cheap and ubiquitous internet access services could reduce the need for universal service subsidies on communications. The framework I outline below aims to suggest a way to balance the need for regulation with allowing decentralized creativity in the context of a complex adaptive system.

\section{The InTERnet/WeB: CHARACTERISTICS, PARAdigmS AND METAPHORS}

\section{A. Characteristics of the Internet/Web}

Three attributes of the internet/web are particularly relevant to the current discussion: it is modular, decentralized, and changing rapidly.

\section{Modularity}

A big change in communications systems in the last fifty years has been the conversion of centrally controlled, tightly controlled "hierarchical" systems to more open, distributed, "modular" systems. Internet /web functionality is built up out of substitutable and separable pieces known as modules.

A module is an interchangeable part of a larger collection of components that delivers a user experience. Modularity is the design philosophy that builds functionality out of partial, separable and substitutable components, the modules. Modules are:

- Partial. A module on its own cannot provide a complete user experience; it's a sub-set of the entire thing. The end user needs to assemble two or more modules to create the result they seek, like combining a local and long-distance phone service provider in the US.

- Separable. A module is self-contained and detachable, eg, a web hit counter or other web plug-in can be removed without affecting functionality of the rest of the page.

- Substitutable. A module can be replaced by another, equivalent one from another supplier, like replacing one web browser by another one.

Substitutability requires some public disclosure of the interface between modules. This leads to the Farrell \& Weiser (2003) definition: "Modularity means organizing complements (products that work with one another) to interoperate through public, nondiscriminatory, and well-understood interfaces." Note, though, that substitutability is not a sufficient condition; it presumes that an architecture of separable and partial pieces already exists.

Examples of modules include network connections like a wired Ethernet connection or a cellular data service; directories, from the DNS to sites like alluc.org that organize links to other resources; web browsers; voice over IP (VoIP) functionality; an AdSense, MySpace or Facebook plug-in on a web page; and a pay-per-view subscription to a video channel that can be delivered via cable or satellite.

Some user-facing modules have enough heft to qualify as applications, though in a modular world they often are built out of modules themselves, and may host other modules. MySpace or Netvibes are applications, but they plug into various browsers, and their functions are extended by other plug-in modules.

Many systems at a given scale may use the same sub-system at a lower scale; the topology is a web, not a tree. For example, all applications on a given machine may use the Windows operating system and DSL connectivity; similarly, TCP/IP etc. protocols underpin broadband connectivity 
provided via DSL, cable modem, or Wi-Fi. (See Figure 3: The Layers Model as a set of hierarchically nested systems in Section V. D below for an example.)

Modularity makes regulation more difficult because the resulting number of industry players is larger, and more diverse, than before. In the days of the Bell System, a small elite that spanned the regulatory and operational divide could quickly agree what had to be done, and how. For example, system engineers might instinctively cooperate with law enforcement to provide surveillance even when the statutory situation was vague. ${ }^{9}$ There are many more points to monitor on the internet these days, and the engineers are not all cooperative; a third of them are not even Americans. ${ }^{10}$ Rules that used to be unwritten now have to be codified, with all the political infighting and unintended side effects that this entails. ${ }^{11}$

A particular user experience can be created using different sets of modules, and the same module can be part of many distinct experiences. For example, web video can be consumed through a media player (eg, iTunes), a web site (eg, YouTube), or a video plug-in on a third party site; and a VoIP module can provide real-time voice capability for both a telephony service and a video game. It is therefore difficult to base regulation on modules, in the way that regulation used to be based on industry verticals, technology architecture, or market definitions. ${ }^{12}$ It's also hard to use modules to define markets, since firms or consumers can mix and match the modules they use to create a service, potentially working around a provider with market power. Cowhey, Aronson and Richards (manuscript) argue that modularity will reduce antitrust concerns on the internet/web. However, the scrutiny of the Google/DoubleClick acquisition and Google/Yahoo partnership shows that bottlenecks could arise in the Web 2.0 world, too. ${ }^{13}$

\section{Decentralized self-organization}

Implementation and control of the internet/web is decentralized. In engineering terms it is a network of networks, and thus a patchwork of autonomous institutions. While circuit-switched telecommunications had a small number of providers, and often a monopoly, there are tens of thousands of internet entities. ${ }^{14}$

Content is created at the edges of the network, by a multitude of autonomous agents. Some providers are large companies, but a huge amount of content is now created by individuals; the distinction between producers and consumers is being blurred. For example, it is reported that

\footnotetext{
${ }^{9}$ As they seem to have done after 9/11 for the NSA, see eg Eric Lichtblau, "Senate Approves Bill to Broaden Wiretap Powers,” The New York Times, July 10, 2008. Available: http://www.nytimes.com/2008/07/10/washington/10fisa.html

${ }^{10}$ Data compiled by NationMaster.com from the CIA World Fact Book indicates that $60 \%$ of internet hosts were in the US in 2007. See http://www.nationmaster.com/graph/int_hos-internet-hosts, https://www.cia.gov/library/publications/the-world-factbook/fields/2184.html

${ }^{11}$ Jonathan Grudin (personal communication) observes that technology unmasks inconsistencies between rules and practice. Rules such as laws, policies, procedures and norms enshrine how we believe people should, and conventionally do, behave. In practice, there are many rule violations, and cases of uneven enforcement which lead to the "right" outcome when rules are "obviously" inapplicable. As technology is more deeply integrated into lives, it exposes ever more deviations between rules and practice.

12 The "silo", "layers" and "EU” approaches, respectively.

${ }^{13}$ See eg http://news.cnet.com/8301-10784_3-9890858-7.html, http://news.cnet.com/8301-1023_3-9989478-93.html

${ }^{14}$ Faratin et al. (2007) report over 26,000 interconnecting entities on the internet, with a growing diversity of interconnection contract types. There are 60,000 interconnection arrangements, and relationships have broadened from either peering or transit to complex blends, like paid peering and partial transit.
} 
uploads to YouTube reached 200,000/day at the end of 2007. ${ }^{15}$ There were 10,000 Facebook applications in December 2007. ${ }^{16}$ Increasing numbers of video blogs approach or meet the standards of broadcast TV. ${ }^{17}$

The internet is not only decentralized; the boundaries between systems are porous, as illustrated by jurisdictional arguments that cross boundaries both physical (between countries) and conceptual (between the physical world and “cyberspace”).

\section{Change and Diversity}

The internet/web is diverse in very many ways: it is both infrastructure and experience; both network and conversation; a marketplace and a community; and simultaneously a medium, a tool, a place, a conversation, a system, and a mirror.

The decentralization of the internet/web increases its diversity. There are many uses and stakeholders, eg, commercial, academic, civic, governmental, which leads to conflict. Geographic diversity is particularly troublesome: even though the network crosses borders and jurisdiction is ambiguous, every state wants to impose their own norms on their citizens even if it has extraterritorial impacts.

The modularity and decentralization of the internet/web drives constant change. The internet/web is a relatively new phenomenon, with many characteristics still unsettled. Business models, governance, usage patterns, usage norms etc. are all rapidly changing. It is nowhere near equilibrium. Many parts of the industry are changing much faster than the regulatory process.

Still, the internet/web is growing up. The flux will subside, though at different rates for different attributes. It is reasonable to assume, though, that it will take a very long time for the pace of change to settle back to the leisurely pace of the mid- $20^{\text {th }}$ century, if it ever does.

\section{Historical context}

The changes described above have been driven by a series of innovations, notably services built out of software, exponential improvement in hardware performance, standardized streams of data and meta-data carried on global broadband networks, and the flourishing of a world of intangibles.

Society is moving from a corporate, mass-production economy to a knowledge-based information economy. The old industrial economy started around the time of the Model T Ford in 1908. It had its heyday in the post-World War II decades. The FCC is rooted in this era; the first Radio Act was promulgated in 1912, and the first Communications Act in 1934. The FCC was invented to regulate an old world, industrial economy. Comparing to the three characteristics listed above, Old Telecoms is characterized by

- Vertical integration. Both the production and distribution chains were tightly linked, and often wholly owned by one enterprise. It was easy to regulate one part of the industry and affect outcomes in another part, eg, regulating owners of TV studios and antennas to deliver "public interest" content, or regulating telcos to deliver universal service.

\footnotetext{
${ }^{15}$ YouTube Editors, February 16, 2006, http://www.youtube.com/blog?entry=jNQXAC9IVRw. Reuters, July 16, 2006, http://www.usatoday.com/tech/news/2006-07-16-youtube-views_x.htm. A blog post in November 2007 reports 200,000 uploads per day: http://blogs.pcworld.com/communityvoices/archives/2007/11/when_will_youtu.html

${ }^{16}$ http://facereviews.com/2007/12/05/facebook-applications-break-10000/

${ }^{17}$ See eg http://dailybuzz.mobuzz.tv/, http://cnettv.cnet.com, http://geekbrief.tv/
} 
- $\quad$ Rigid organization. There were few producers, in a one-to-many relationship with welldefined consumers. The arrangements were very stable - indeed, some were de facto or de jure monopolies.

- Slow change. Systems didn't change quickly, since they were built in hardware, not software. Mass markets had a great deal of inertia, and rapid product innovation was neither necessary nor desirable.

The information economy got going around the time of the first Intel microprocessor in 1971, and hit its stride in the mid-90s. It's no surprise that we're in need of a regulatory rethink. The Telecommunications Act of 1996 was the last gasp of the old era, and the new regulatory era has not yet seen its first major milestone.

\section{B. Regulatory Paradigms}

Telecommunications policy in the $20^{\text {th }}$ century was organized around a division between service categories (Werbach 2002). These categories, often called "silos", include wire-line telephony, radio communications including over-the-air television, and cable television. A service is a combination of delivery medium, implementing technologies, user experiences, business model, and market. Historically the services were distinct from each other on all counts. A classification by any of these criteria served to distinguish between the others, so that a classification by service was equivalent to a classification by transport medium.

The internet broke down the distinctions between the categories. Transport facilities, originally classified differently because of the analog services associated with them, could carry the same digital service: for example, internet access can be offered over cable, wire-line telephony, or cellular.

The challenge of constructing a new framework was taken up in the late 1990s in a Green Paper by the European Commission (1997). In examining options for future regulation, the Green Paper noted that one of the leading alternatives to the then-current approach was "to shift away from a vertical model of sectoral regulation and towards a horizontal approach that seeks to distinguish between the network or transmission layers within converging sectors and the services carried over those networks”. The EU Framework Directive (European Parliament 2002) that followed set out to address competition and user interests in a consistent manner across all kinds of infrastructure in a consistent way. ${ }^{18}$ To this end, it created technology neutral categories: Electronic Communications Networks (transmission infrastructure), Electronic Communications Services (conveying traffic; content services are excluded), and Associated Facilities (a grab-bag of enabling technologies). There was a presumption that companies that do not have significant market power will bear minimal regulation.

The approach that emerged in the United States also addressed the convergence of transport technologies, but went further to create a "layers model" that urged policy makers to reformulate communications policy around the technical architecture of the internet (Werbach 2002, Solum \& Chung 2003, Whitt 2004). Industry-based distinctions would give way to different treatment for layers corresponding to the internet's implementation of packet-switched networking. The layers model has been criticized for not being technology neutral, for understating the importance of market power analysis, for difficulties in demarcating layers, and for inhibiting price discrimination (Marcus 2006, Odlyzko not dated).

\footnotetext{
${ }^{18}$ See http://icttoolkit.infodev.org/en/PracticeNote.514.html for a summary.
} 
The silo and layers models are attractive because they're one-dimensional: a single parameter serves to distinguish between categories. In the silo model, the parameter is end-user service (broadcast TV, telephony, satellite communications, etc.); in the layers model, it's the degree of abstraction above the physical transmission of data. However, since both end user experience and technical architecture are important distinctions, neither silos nor layers are sufficient on their own. Near to the physical layer, networking architecture dominates, and end-user experiences can be largely ignored; but from the user perspective, watching video is different from voice communications even if both are implemented via packet switching. Layers are helpful low in the stack, but the particular public interest mandates applied to TV are different from those on telephony, and so service silos can't be ignored. The argument is at its most complex in the middle of the network stack, where the two models blur into each other.

An enhanced technology-centered regulatory perspective that reflects the complexity of the internet/web would have to be based on modules. However, the multiple ways in which modules can be used to build services make this very difficult (cf. the section on Modularity above). My working hypothesis, not addressed further in this paper, is that regulation in the age of modules should apply to the capabilities that are exposed, not the means by which they're delivered; for example, if a user experience is functionally and socially equivalent to traditional telephony, access to emergency services should be provided regardless of implementation. Matters are complicated, though, by the fact that the context in which a capability is delivered makes a difference; for example, a voice chat on X-Box Live during a game is different from voice communications module embedded into an employee's work desktop. Modules further complicate matters when an end-user builds up an experience by using modules from different providers. For example, imagine a visually disabled person builds a portal on Netvibes with newsfeeds from various web sites, an IM plug-in from one player, and a voice module from another - who's responsible for delivering accessibility functions?

The alternative proposed here is a principles approach that is technology- and industry-agnostic.

\section{C. Current Metaphors}

Metaphors use a familiar domain to explain a less familiar one. Conceptual metaphors make systematic use of the structure of one conceptual domain to reason about another (Lakoff and Johnson 1980). Science often advances by working out the implications of a metaphor mapping. Mary Hesse (1966) has explored the role of metaphor in physics in great depth, and historians of economic thought have argued that classical physics lies at the heart of neoclassical economics (Mirowski 1989, Klamer \& Leonard 1994). A great deal of metaphorical thinking is also found in the law (Schlag 2002, Winter 2003).

Metaphors are mental models, and like any model they suppress some aspects of a situation while highlighting others. A "good" metaphor is productive: it is intelligible, reliable regarding salient considerations, and inspires effective solutions.

Since the internet/web is a relatively new phenomenon, metaphors that for it abound. A slashdot discussion during April 2007 about defining the Internet gave many technical definitions, but also many metaphors: highways, trains, telephones, and the mail. ${ }^{19}$ The metaphor of systems and networks was very common, and there were a few references to its social aspects. Anthropomorphism was pervasive: computers talking to each other, sending messages, sharing information with each other, etc.

\footnotetext{
${ }^{19}$ http://ask.slashdot.org/article.pl?sid=07/04/26/0535200
} 
A web search to find ways of describing the internet/web yielded a similar set of terms. ${ }^{20}$ Communications infrastructure is the most common overarching category: highway, medium, network, or pipe. This concept also informed one-off metaphors like "post office". Transport came into play in the vehicle metaphors, like "car". The other common thread was that the internet/web is some kind of place. There was quite a lot of "information repository" language, particularly references to libraries and databases. A repository is a tool, and one might say that this was the third common theme: the internet/web as a means to an end. There were a few references to the commercial aspects, eg, market, way to make money. Other less-frequent but not uncommon images were the internet/web as a social activity (eg, a conversation), as a mirror of society, and as a system.

Susan Crawford (2007) contrasts the very different ways that "Engineers", "Telcos", and "Netheads" define the Internet; each has their own metaphor. The founding engineers see the internet as a logical architecture. The incumbents (telcos) see it as privately-owned pipes that connect consumers to content. The internet futurists conceptualize it as standards and relationships that give rise to persistent social worlds. These three ways of thinking about the internet can be seen as views of the same system at different resolutions:

- $\quad$ logical architecture (the view of the founding engineers) focuses on the protocol scale;

- $\quad$ privately-owned pipes that connect consumers to content (the view of the incumbent network operators) focuses on the network scale;

- $\quad$ the web as relationships that lead to society (the view of the "netheads") focuses on the human scale.

This nesting also partially explains the wide diversity of metaphors for the internet/web. For example, the "internet/web as communications infrastructure" metaphor focuses on the network and protocol scales, while the "internet/web as place" and "internet/web as tool" metaphors focus on the tool scale.

All these metaphors help policy makers develop mental models that inform action. Like all models, they have strengths and weaknesses. Their key strength has been the familiarity of images of place and references to tools, and the credibility engendered by references to engineering. On the other hand, most engineering analogies do not capture the contingency and uncertainty of the internet/web's operation in practice, as seen in hacker/sysadmin arms races, the disruptive potential of new technologies like Napster and BitTorrent, and cultural phenomena like Wikipedia and MySpace. Place and pipe metaphors represent the internet/web as a static container for action, obscuring the interplay between the technology, policy, commerce and users such as that at play in the network neutrality debate. The view of the internet as a conversation, on the other hand, privileges social dynamics over engineering and commerce, again suppressing the mutual dependence of politics and process.

A more integrated metaphor that combines engineering, culture and politics is required. Such a framework will be introduced in the next two sections.

\footnotetext{
${ }^{20}$ Google search 17 Aug. 2007 for phrases "internet is a”, "internet is like a”, "the web is a", and "the web is like a". I used "the web is...” since simply searching for "web is a ...” throws up many results for the semantic web, which is a specialization I want to ignore in this case. Only terms that occurred more than twice in the first ten pages of search results were considered. The internet was described as highway, library, marketplace, medium, mirror, network, pipe, place, repository, social activity, system, tool, and vehicle. The list for the web was largely the same, though the metaphors 'network' and 'pipe', often used for the internet, did not appear. The 'graph' metaphor, which wasn't used for the internet, was used quite often for the web.
} 


\section{THE FORESTRY METAPHOR}

A forest is a particularly illuminating example of a managed complex adaptive system since the analogies with the internet/web are straightforward. This section draws out these correspondences in order to make the case that lessons from managing forests and other large ecosystems can provide guidance in communications policy.

Most of the action in both forests and communication systems happens spontaneously, but management of the system plays a critical role in both cases. The existence and form of the forest is the result of intentional human action, subject to the vagaries of exogenous, unpredictable influences such as weather, pests and politics. In the same way, the internet/web is more than just an autonomous market/culture complex, where companies, customers and citizens interact. While internet/web innovation is driven by entrepreneurs and technologists with their own agendas, it is shaped by government decisions.

A forest is neither pure nature nor pure culture; it is nature in the service of culture. Likewise, the internet/web is neither pure technology nor pure politics. The communications market place, left to its own devices, will not automatically provide all needed social goods, any more than nature, left to its own devices, will necessarily rebuild a flattened forest as an idyllic stand of pines rather than an overgrown bramble patch. In both cases, humans have to take guiding decisions.

Managed ecosystems vary in scale from window-sill planters to national forests, and in governance from personal to public to commercial. Some forestry objectives are utilitarian, and others aesthetic; some see forests as primarily productive and others cultivate them for pleasure. In the same way, some data networks range in size from personal and corporate to trans-global. Objectives range from personal and organizational productivity, to frivolous entertainment and political advocacy.

Just like the internet/web, managing a forest is often an exercise in frustration; one can never know precisely how things will turn out. Animals that thrive in one part of a park inexplicably disappear in another. Plagues of pests and disease appear unexpectedly; unexpected consequences abound. Policy makers and regulators are like foresters and park rangers; while they have a crucial role and great responsibilities, their reach is limited.

The forest manager is largely irrelevant and invisible to the plants, animals, bugs, microbes that do the work in a forest. They experience the ranger as part of their context, and often as a force to be contested or co-opted. However, if one generalizes the definition of "manager" to be any organism that attempts to shape outcomes in its environment in order to further its own agenda, then every plant and animal - and groups of them acting in concert - are managers each at their own scale. For example: mature forests moderate local climate to their own advantage; some trees poison other plants around them; and earthworm casts change the make-up of soil, improving the situation for themselves and others. There are many managers, not just at various scales, but also at a given scale. Competing organisms contend towards contradictory ends. In forestry this is most obvious at the regional management level, where there are many agencies with overlapping briefs that are responsible for the same geography. Who supervises the supervisor? The answer is that the forest keeps the forester honest. It sets the constraints of what will grow and what will not, and reminds the park manager of his or her limitations, often mercilessly.

In the same way on the internet/web, every sub-net, every service, and every applet work to further their own agenda; they garden their local environment. Each competes strenuously with its peers. Policy makers are ignored if possible, and tolerated if necessary. Governance decisions are contested. Participants have different interests, and want different outcomes.

Elk, birds, beetles, and seeds don't respect national borders. A forest is embedded in a larger regional system, and its boundaries are porous. Tension with neighbors is inevitable. There is 
management at many scales, from a gardener's decision about what variety of tomato to plant for next year, to state-wide prohibitions on planting noxious weeds, to cross-border initiatives to manage migrating birds. ${ }^{21}$ Different gardens have different sets of rules. By the same token, different spheres of the web will need different rules. There are conflicts over cross-border jurisdiction both in forestry and on the web. ${ }^{22}$

The ecosystem management metaphor provides a way of understanding the change in communication systems over the last few decades. The old silos of traditional communications regulation resemble commercial farming. There are a limited number of well-defined fields, each with its own crop: corn, wheat, beans, vegetables, and so on. It's clear who is responsible for the farm, and the measures of success, like bushels per acre per dollar of inputs, are well defined. Today's internet/web is more like a planet-wide patchwork of parks and forests, making internet policy is like public forestry, or gardening on a global scale. Control is decentralized, and there is a great deal of variety. There are many competing uses and users, from logging to pleasure to ecological services. Given this pluralism, success metrics are ambiguous at best.

The metaphor of the internet/web to a forest is necessarily incomplete, like any analogy. Different perspectives of the internet will each reveal truths that are neither entirely independent nor entirely compatible. The forest metaphor does, though, capture the web's complex system dynamics in a familiar and reliable way. ${ }^{23}$ It fits in the tradition of other place-based metaphors for the web (as a building, market, library, or public venue). There is a resonance with tool metaphors, since gardens are a means to an end, whether pleasure or production. The link to the "internet as communications infrastructure" metaphor is less direct, but they don't contradict each other. It is therefore intelligible. Finally, the forestry/complex systems analogy also readily suggests policy solutions, as will be demonstrated below. One might therefore qualify the metaphor as "good".

Table 1: The internet/forest metaphor map

\begin{tabular}{l|l}
\hline Forest & Internet/web \\
\hline plants animals and other organisms & firms, users, citizens \\
forester & policy maker \\
cultural expectations and human actions & $\begin{array}{l}\text { social pressures and policy decisions } \\
\text { shaping the internet/web }\end{array}$ \\
shaping a park system & $\begin{array}{l}\text { technology, engineering, commerce, and } \\
\text { user activities }\end{array}$ \\
niological and physical processes & national internet/web \\
gardional forest and park system & home network \\
managed ecosystems world-wide & global internet/web
\end{tabular}

\footnotetext{
${ }^{21}$ See, eg, Debora L. VanNijnatten, “Towards Cross-Border Environmental Policy Spaces in North America: Province-State Linkages on the Canada-U.S. Border”, for the findings of a 2005 survey of environmental linkages along the Canada-US border. Available at http:// ejournals.library.vanderbilt.edu/ameriquests/include/getdoc.php?id=366\&article=62\&mode=pdf

${ }^{22}$ In the web case "cross-border" refers to bother geography and functionality, eg, between what people do in synthetic worlds and games, vs. content posted on Youtube, vs. stuff that's done in email.

${ }^{23}$ It is worth recalling that the concepts "system” and "ecosystem” are also metaphors. An ecosystem, and even more a managed ecosystem, is a human construct imposed on multifarious and slippery reality. It's a just a model; and all models are incomplete.
} 
fences

major crops and fields in large farms borders

silos of traditional communications

\section{Other ecosystem metaphors for the internet/web}

Steven Berlin Johnson likened the web to a rain forest. ${ }^{24}$ He argued that the difference between Web 2.0 and previous technology generations is like the difference between a rain forest and a desert. Information absorption efficiency of Web 2.0 is dramatically higher, just as rain forest is more efficient than a desert at using energy because there are so many organisms exploiting every tiny niche of the nutrient cycle.

Johnson's rain forest metaphor is related to the industrial ecology metaphor. Industrial ecology sees industrial systems as being part of the biosphere, and focuses on finding solutions to ecological problems. ${ }^{25}$ The ecosystem analogy has been taken up as a model for business, particularly in the information technology industry (Iansiti \& Levien 2004). However, it is not clear to what extent the metaphor should be taken seriously as a model of the system. ${ }^{26}$

A group of business strategists, regulators and academics that met at Rueschlikon in June 2007 seized on the metaphor of gardening as a model of how governance of information in a global economy might deal with the difficulty of predicting how the system will evolve and change (Cukier 2007). Participants variously observed that regulatory perspectives need to be reconstructed as a game played by agents in a network; that rule-sets ought be defined at the lowest feasible level of granularity, with the recognition that that significant emergent properties exist; that when innovation blossoms, it is usually unanticipated; and that the conflict of values between those who see weeds or wilderness in the same plot of land.

\section{INSIGHTS FROM MANAGING COMPLEX ADAPTIVE SYSTEMS}

This section sets out to show that forestry, managed ecosystems, and complex adaptive systems in general, provide tools for thinking about internet policy. The four Ecosystem Management Principles of flexibility, delegation, big picture and diversity will be derived in Section VI.

It should not be surprising that the forestry metaphor makes sense for the internet, since both ecosystems and the internet/web can be modeled as complex adaptive systems. Other metaphors that link managed adaptive systems to the internet/web may work just as well, and may be more

\footnotetext{
${ }^{24}$ http://www.stevenberlinjohnson.com/2005/10/why_the_web_is_.html

${ }^{25}$ For a brief history of the field, see http://www.is4ie.org/history.html. See also http://en.wikipedia.org/wiki/Industrial_ecology. Contrary to this view, ecologist John Harte argues that while ecological sustainability practices under the banner of "industrial ecology" are worthy and important, they do not mimic the way natural ecosystems work; see "Business as a Living System: The Value of Industrial Ecology A Roundtable Discussion,” California Management Review (Spring 2001).

${ }^{26}$ Iansiti \& Levien note that "We are not arguing here that industries are ecosystems or even that it makes sense to organize them as if they were, but that biological ecosystems can serve as a source of vivid and useful terminology as well as provide specific and powerful insights into the different roles played by firms.” (Authors' italics.) As this quote suggests, there are a variety of problems with the metaphor as a model, ranging from mismatches between the scale of biological and business ecosystems, the absence of conscious goal-setting in biological systems, and the qualitative difference between energy and information flows. ${ }^{26}$ Further, metaphors of business as ecosystem generally do not treat policy making as a component of the system, but focus rather on interacting firms as a web of organisms in a "state of nature". However, they demonstrate the value of thinking about the web in terms of familiar biological images. For a blog-level discussion of weaknesses of the business ecosystem metaphor, see my http://deepfreeze9.blogspot.com/2007/06/eco-mumbo-jumbo.html, 27 Jun. 2007.
} 
intelligible to certain audiences; examples include fisheries management, transnational enterprises, and the network layer of the internet.

\section{A. Definitions}

A "system" is an organized collection (frequently, a self-organized collection) of elements that acts over time to produce reasonably predictable outcomes. Each element affects the whole, and the whole influences the behavior of the parts. The parts cannot be understood only by studying the whole, and the whole has properties that are not inherent in any of the parts. Systems self-organize many of their interactions without outside intervention, and their characteristic structural and behavioral patterns are mainly a result of interaction between the sub-systems.

There are many schools of "system thinking". ${ }^{27}$ In general, this approach shifts the emphasis from an analysis of separate parts to their organization, and from static analysis and description to dynamic activities and processes.

Ervin Lazslo (1996:17) contrasts the traditional reductionist goal of finding commonality in substance with the systems theory goal of seeking universality in organization. The diversity of the internet/web means that there's little or no common substance, but there is commonality in organization at various layers: different connected networks use similar protocols; social networks organize themselves in similar ways using different media; communications of widely different types (IM, SMS, email) serve the same social purposes. A systems approach thus seems better suited to analyzing the internet/web than a reductionist one, which posits that reality can be reduced to a number of indivisible basic elements, that phenomena at higher levels can be fully explained in terms of ones at lower levels, and that qualitative properties can be reduced to quantitative ones (Skyttner 2001:13).

A "complex adaptive system" is a collection of interacting adaptive agents. ${ }^{28}$ The term is often associated with a research field that developed in the 1980's at the Santa Fe Institute. Attributes that distinguish complex adaptive systems from other collections of agents include self-similarity, complexity, emergence and self-organization. Complex systems show multiple, non-linear interactions among components at different levels of aggregation. Such systems are not decomposable into sub-processes - say, economic, technological, or political - that can be understood and managed in isolation. Complex systems typically have a nested hierarchical structure, with interactions across the levels (scales) of the hierarchy. Processes respond non-linearly to inputs; there is a mix of fast and slow processes; time lags play a critical role; outcomes are path dependent; and components adapt to disturbances through feedback loops. ${ }^{29}$

Carlson and Doyle (1999) focus on systems that have self-dissimilar internal configurations; this contrasts with the self-similarity that is the basis for a great deal of the earlier literature. These highly organized yet non-generic structures are models for many systems, from biological organisms to technological artifacts. ${ }^{30}$ Carlson \& Doyle argue that such complexity, which they call "highly

\footnotetext{
${ }^{27}$ For surveys, see Richardson (1991), Skyttner (2001) and http://en.wikipedia.org/wiki/Systems_theory

${ }^{28}$ http://en.wikipedia.org/wiki/Complex_adaptive_system

${ }^{29}$ For a discussion of these effects in a biological setting, see Walker \& Abel 2001; for a commercial setting, see Sterman 2001; for applications in economics, see Beinhocker 2006

${ }^{30}$ A commercial jet aircraft is an example of a highly organized system that displays robust yet fragile behavior. It is a complex system composed of many, diverse sub-systems, each with many components. The plane is embedded in a larger network of air transportation, which includes systems for pilot training, pre-flight checks, regular maintenance, and regulatory supervision that is intended to catch failures in the plane system and prevent a catastrophic collapse. This system in turn is embedded in a larger economic and social system. Over time the supervisory system also becomes more complex, with periodic adjustments and simplifications. The risk of cross-scale failure increases as
} 
organized tolerance”, necessarily emerges when systems are engineered (or evolve) to operate in a wide variety of situations. Unfortunately, this complexity can also lead to catastrophic failure; they thus describe these as "robust yet fragile" systems.

The study of complex systems yields four attributes that inform the policy principles that are proposed below: incomplete knowledge, cycles and transitions, hierarchy and cross-linking, and surprise and novelty.

\section{B. Incomplete Knowledge}

It is not possible to set up analytical models for complex systems. Any model that purports to capture the behavior of a system necessarily under-represents it (Bankes 2002). No model less complex than the system itself can exactly, and in detail, forecast its behavior. It's a trade-off: analytical tools work either for complicated systems that are relatively predictable, or for simple systems that are uncertain, but not for systems that are both complex and uncertain. ${ }^{31}$

Conflicting explanations compound a deeper issue: the lack of agreement on the problem at hand. Many policy debates entail deep uncertainty, defined by Lempert et al. (2002, 2004, following Kenneth Arrow) as the condition where the decision maker does not know, or multiple decision makers cannot agree on, the system model, the prior probabilities for the uncertain parameters of the system model, and/or the value function used to rank model outcomes. Deep uncertainty resembles Knightian uncertainty, where randomness cannot be expressed in terms of specific mathematical probabilities. Rittel and Webber (1973) coined the term "wicked problems" to describe a similar set of challenges in making social policy, including the absence of a definitive problem formulation; the lack of a stopping rule or an ultimate test of a solution; and the lack of an enumerable set of potential solutions.

Systems thinker David Weinberg posited the "complementary law", which states that different perspectives on a system will reveal truths regarding that system that are neither entirely independent nor entirely compatible (Skyttner 2001:92). In other words, a complex system has many distinct but equally valid descriptions. This means that conflict in policy making is unavoidable. Since people have different perspectives, they will form different assessments of the situation and varying valuations of desirable outcomes.

The many ways to describe the internet/web are instances of the complementary law at work. ${ }^{32}$ They include the many metaphors discussed above, as well as the variety of current and proposed regulatory frameworks, such as the industry/technology segmentation of titles in US communications statutes, the technology neutral approach of the EU Framework Directive, and the various layers models.

The incompleteness of any model of a complex system and the necessity for complementary perspectives suggest that policy makers take a big picture approach: a broad view of how problems might be solved. The deep uncertainty about these systems also implies the need for flexibility: since one cannot be sure of either the problem or the best solution. (Big Picture and Flexibility are two of the four principles that will be discussed in more detail below.)

systems at different resolutions sync up; this might happen in the airline business if slowing growth at Boeing and Airbus prompts them to get into the pilot training and plane maintenance businesses in a big way.

${ }^{31}$ There are, though, pockets of predictability in complex adaptive systems - see Andersen \& Sornette 2005.

32 This means that the approach of this paper is also necessarily incomplete and doesn't replace others. 


\section{C. Cycles and Transitions}

Complex adaptive systems can have many stable states, shifting from one to another from time to time. Sometimes they return to states previously visited, showing cyclical behavior. In other cases, a system might flip to an entirely new equilibrium state, unlike any previously occupied. The route that a system takes between these states is a function of its history: path dependency is a classic trait of complex systems, and is well known in economics and planning. ${ }^{33}$

Holling's panarchy theory (Gunderson \& Holling 2001) proposes that biological systems exhibit a four-stage cycle of growth, maturity, collapse, reorganization, and back to growth. ${ }^{34}$ This adaptive cycle operates at different rates in each of a system's many spatial and temporal scales. During the growth stage, there is rapid colonization of recently disturbed areas, for example after a forest fire or windstorm has removed large amounts of biomass in a forest. The connectedness between organisms is low, which leads to high resilience; the loss of one species doesn't lead to the loss of another. As the forest matures it moves into the maturity phase of the cycle, dominated by the accumulation of material. The network of connections between biomass and nutrients becomes increasingly tight, and fragile; every niche in the forest is filled, and every resource is used. Organisms become much more interdependent; food chains become dense and interconnected. The maturity phase is followed by a dramatic collapse phase, triggered in a forest by fire, drought, insect pests, etc. A lot of energy is unbound, and networks are broken up. This sets the scene for the fourth phase, reorganization: opportunistic species that have been suppressed by the stable configuration of the maturity phase move in. This is a period of innovation and restructuring, laying the groundwork for a return to another growth phase.

Collapse is inevitable, and necessary for renewal in ecosystems. Since complex systems operate at many concurrent scales, however, the collapse phase at one scale need not trigger the collapse of the entire ensemble. A system is robust if the cycles at different scales are not in sync, eg, a forest is relatively robust against catastrophic fire damage if its peak maturity (and thus susceptibility to fire) doesn't coincide with a temperature peak in the regional climate cycle.

Jain and Krishna explored the punctuated equilibrium of biological ecosystems and found find repeated rounds of crashes in which several species get rapidly depopulated, followed by recoveries (Beinhocker 2006:174). The system starts in a random phase, where it burbles along without much structure. An innovation suddenly sends the system into a growth phase; there's an innovation cascade that eventually flattens out into an organized phase that is highly structured with keystone species at critical points in the interaction web. When an innovation or random change hits a keystone species ${ }^{35}$, the network crashes in a wave of extinctions, leading to a restart in the random phase.

One can map the Holling adaptive cycle to phases in industry development. When technology or some other disturbance opens up a new market, there is a growth phase where economic connectivity is low; supply chains are rudimentary. ${ }^{36}$ Consolidation follows: a maturity phase where revenues grow steadily and everybody finds their niche and stops competing at the margins.

\footnotetext{
${ }^{33}$ See eg Arthur (1994), Rittel \& Weber (1973)

${ }^{34}$ They use the terms exploitation, conservation, release, and reorganization for the four stages.

${ }^{35}$ Keystone species: an organism whose role in its ecosystem is much larger than would be expected from its abundance

${ }^{36}$ Even though economic connectivity may be low, social networks may be rich. Peter Haynes points out that most innovation takes place in geographical concentrations where there is very high inter-personal connectivity and quick cycle speed (personal communication). Dependencies remain low, though; the failure of one start-up doesn't lead to the failure of a series of others.
} 
Innovation begins to decline. There follows a collapse phase where some disruptor breaks the reigning industry model, and a lot of money is lost. ${ }^{37}$ Revenues drop in the market as previously defined, even though economic productivity continues to grow. New entrants flood in to reorganize the industry, with a boom in experimentation.

Many if not all commodity markets show booms and busts (Sterman 2000, Ch. 20). Economies show all the characteristics of complex adaptive systems. ${ }^{38}$ One can be confident that such phenomena occur in the information and communications industry too. ${ }^{39}$ For example, there were few applications in the early days of mainframes. The numbers grew as the customer base expanded, though most applications were developed in-house by hardware vendors. The introduction of the PC disrupted this pattern, leading to a decline in the relative importance of mainframe applications. At first there were few PC applications, but then VisiCalc provided the breakthrough that led to the explosion of PC applications in the early 90s. The interconnection between applications was low at first, but grew (cf. OLE, and application suites). The advent of the web in the mid-90s decimated the PC application business, going from whole retail stores devoted to software to a single aisle at Circuit City. Egghead was founded in 1984, and closed its retail operations in 1998.

At a deeper nesting in the hierarchy, consider communications services. There was a blossoming of telephone companies in the 1900s-1920s, which ended with the creation of the AT\&T monopoly. This was broken up in 1982, leading to a plethora of competitors, which decreased again to a handful in the early 2000s. ${ }^{40}$

Many complex systems have two (or more) stable configurations. A favorite model of ecologists is lake turbidity, in which biological and political processes are intertwined (Scheffer 1999, Scheffer et al. 2002). The more vegetation in a lake, the clearer the water, that is, the lower the turbidity. As one adds nutrients, eg, from agricultural run-off, turbidity increases as phytoplankton grows. For a given nutrient load, two states are possible within a certain range: low and high turbidity. ${ }^{41}$ However, once a critical nutrient load is exceeded, the lake-bottom plants die off because of lack of light, the water-clearing fauna that depended on them die as well, and the lake flips into a highturbidity state from which it won't recover even if nutrient loads are reduced below the critical point. Nutrients have to be reduced well below the critical point to bring back plant life and switch the lake back to the low-turbidity state. Economic analysis and political pressure tends to drive the system to

\footnotetext{
${ }^{37}$ In Holling's analysis, the mature phase of a forest - the one just before collapse - contains a very large number of species in very many, very specific, tightly interlocked niches. The analogy to business isn't direct; there aren't necessarily many firms at the peak. A large number of interconnected products at the peak may well be internalized to a monopolistic firm. One will see a great deal of diversity and interconnectivity within the firm (eg, feature bloat in Windows and Office) but not in the industry at large. At this point the system is particularly vulnerable to cascading failure, eg, through security flaws, app compatibility or robustness bugs, new market entrants, or antitrust attack.

${ }^{38}$ Samuelson worked on this in the 1930s; Beinhocker (2006) provides a contemporary survey.

${ }^{39}$ Perhaps the internet/web is so recent that it hasn't been around long enough to meander through enough of parameter space. It has mostly been in start-up/growth mode, and may be heading for maturity; the crashes are yet to come.

${ }^{40}$ W. A. Brock (2001) tells the story of AT\&T in terms of the Holling adaptive cycle. After open competition at the beginning of the telephone industry, the Bell System emerged with a dominant monopoly in the late 19th century (maturity). Patent expirations in 1893-94 led to partial breakdown of its monopoly (disruption). This triggered reorganization and a phase of open competition from independent telcos (restart and competition). Around 1907 it started absorbing the independents, evolving into a monopoly again (maturity). An antitrust crisis in 1915-19 led to the creation of a regulated monopoly, which survived into the 80s (rapid disruption, restart and re-consolidation). The court-ordered break-up of 1982 led to a period of renewed competition (disruption, restart, innovation). However, the Telecom Act of 1996 allowed consolidation to restart, and the industry is rapidly maturing again.

${ }^{41}$ This is true within a certain range of nutrients. If the nutrient inflow is very low, one only finds the clear state. If the nutrient load exceeds a critical amount, a lake will always be turbid.
} 
the point where nutrient input from agriculture is high, but just below the tipping point to high turbidity - a point that is unstable to small variations in inputs, which can lead to a rapid transition to high turbidity from which it is costly and politically difficult to return. This is an example of narrow economic efficiency reducing system resilience, a theme that will recur later.

Integration and dispersal of distribution and content production seem to be two stable states in the video business. In the 1980s cable companies used their infrastructure control - and thus, control of access to viewers - to take control of content producers. However, by the 1990s they had started to spin out their media operations (eg, Liberty Media spinning out of TCI/AT\&T in 1991) and are back to pretty much pipe-only operation. ${ }^{42}$ Those who worry about the power of local internet access providers argue that they may be in a position to repeat the integration of transport and content seen in cable systems.

There have been waves of consolidation of control over content in the over-the-air, cable and satellite businesses, not to mention movies. In each case the disrupter (cable vs. broadcast, satellite vs. cable) was blocked by the incumbent as it tried to gain access to content. One might predict that this will happen again on the internet/web as direct-to-PC players try to supply content. This has definitely happened with telcos trying to put together packages for their fiber plays; the Viacom/YouTube litigation may be the rerun on the web.

One learns from ecology that disruptive cycles are unavoidable and indeed healthy since they lead to innovation - they get rid of incumbents for a while and allow new experiments. The trick is to ensure that the collapses are localized. For example, a lot of small, local forest fires keep the litter load down, and prevent massive fires that burn so hot that they essentially sterilize the soil which precludes seed germination and soil health. This suggests that diversity and taking a big picture view, both principles to be discussed below, are useful tools in complex system management. A rigid, unchanging structure is liable to catastrophic collapse, while a more diverse and flexibly managed arranged is more resilient.

\section{D. Hierarchy and cross-linking}

Systems consist of nested sub-systems with linked dynamics at different scales. As a system grows, its complexity increases and a hierarchy emerges. Each level is made up of several subsystems, which can themselves be decomposed. The higher levels control aspects of the lower level sub-systems. However, while the higher layers may be complex, their intricacy is dwarfed by the aggregate complexity of the lower layers (Skyttner 2001:60).

Panarchy theory describes how hierarchies turn into dynamic structures in a biological setting. Natural systems have a variety of time/space scales; for example, a boreal forest has a hierarchy of vegetation ranging from pine needles at the centimeter scale, through tree crowns, stands, and forests, to landscapes at the $1,000 \mathrm{~km}$ scale. Needles come and go on a time scale of less than a year, whereas forests can persist for many centuries (Gunderson \& Holling 2001, Fig 3-8, p. 68). Growth and collapse cycles operate at different rates at each scale.

Cross-scale interactions are particularly important at times of change and renewal. Critical change in one cycle can cascade up to larger and slower scales when they're vulnerable, eg, when a ground fire in a forest spreads to the crown of a tree, then to a patch in the forest, and then to a whole stand of trees before it is finally extinguished. Conversely, renewal at a given level can be

\footnotetext{
${ }^{42}$ See eg Stephen Keating, Cutthroat: High Stakes and Killer Moves on the Electronic Frontier (Boulder: Johnson 1999). David Waterman \& Andrew Weiss, Vertical Integration in Cable Television (Cambridge: MIT Press 1997). Megan Mullen, The Rise of Cable Programming in the United States (Austin: U of Texas Press 2003)
} 
supported by drawing on resources at larger/slower scales, as when a burnt forest draws on its accumulated seed bank and soil nutrients to re-grow.

Figure 1 illustrates the hierarchy in a complex system by showing one simple cut through the system/sub-system nesting for forestry. Each layer is the unpacking of a super-system at the immediately preceding scale; it is like nested matryushka dolls rather than a layer cake.

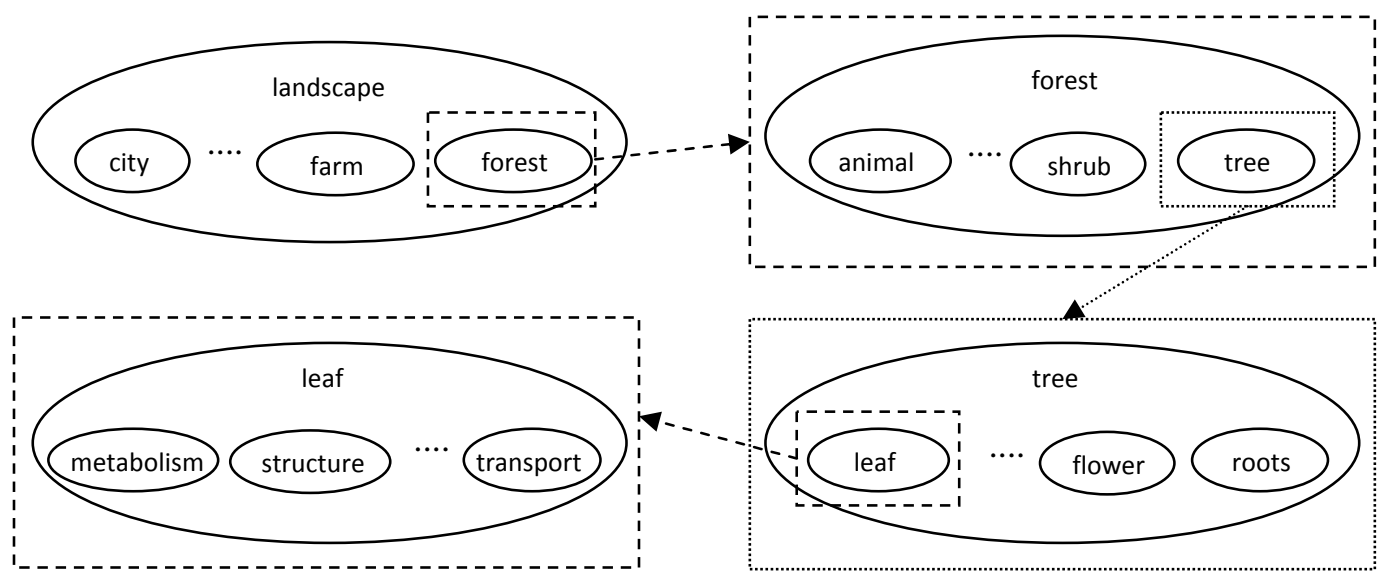

Figure 1: A forest as a set of hierarchically nested systems

The nesting reads clockwise, starting from top left. Many levels in the hierarchy are omitted. Note that sub-systems are re-used, eg shrubs as well as trees have leaves, and elements of leaf structure are also found in flowers. Some levels of the hierarchy, eg, tree stands, are suppressed.

Figure 2 drills down into one nesting sequence for the internet /web in order to illustrate the analogy a forest and the internet/web:

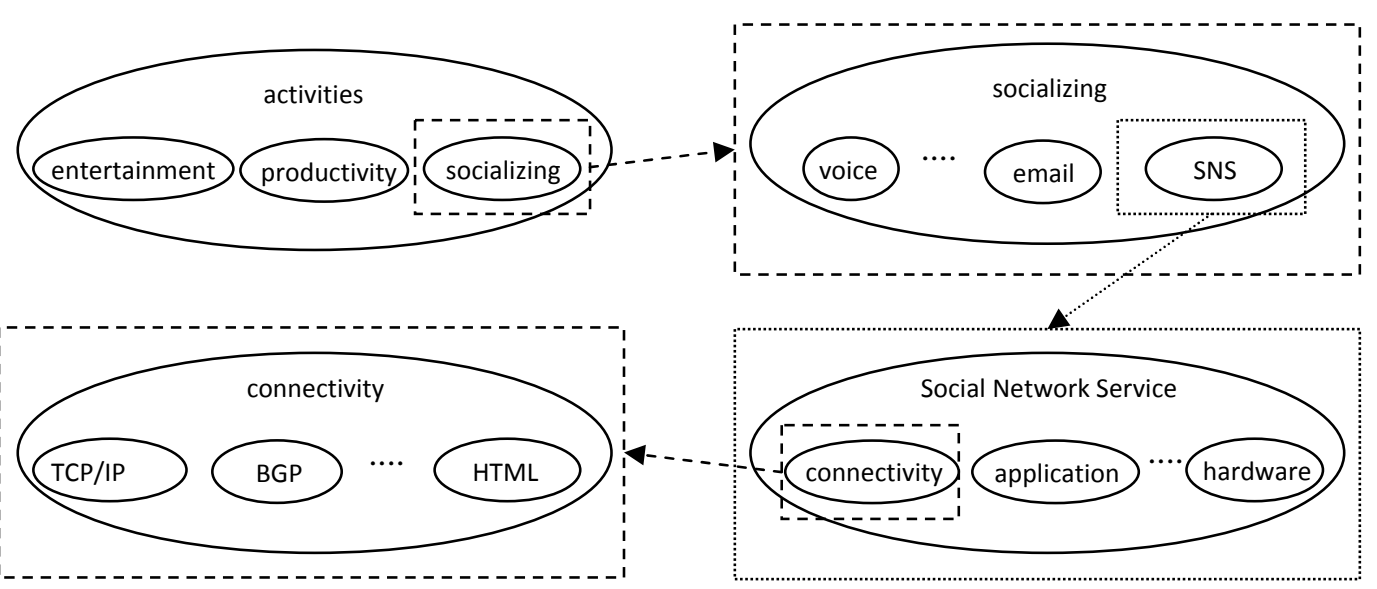

Figure 2: The internet/web as a set of hierarchically nested systems

The nesting reads clockwise, starting from top left. Many levels in the hierarchy are omitted. Note that sub-systems are re-used, eg, connectivity is used by email as well as SNS. 
The hierarchy implicit in a complex system helps to explain why a layers approach can help guide policy. For example, one can represent the four layers of Werbach (2002) or Solum \& Chung (2003) as a sequential unpacking in the same way as above (Figure 3). However, since the hierarchy is in fact a nested encapsulation rather than superposition of layers, separation between layers will be problematic in practice.

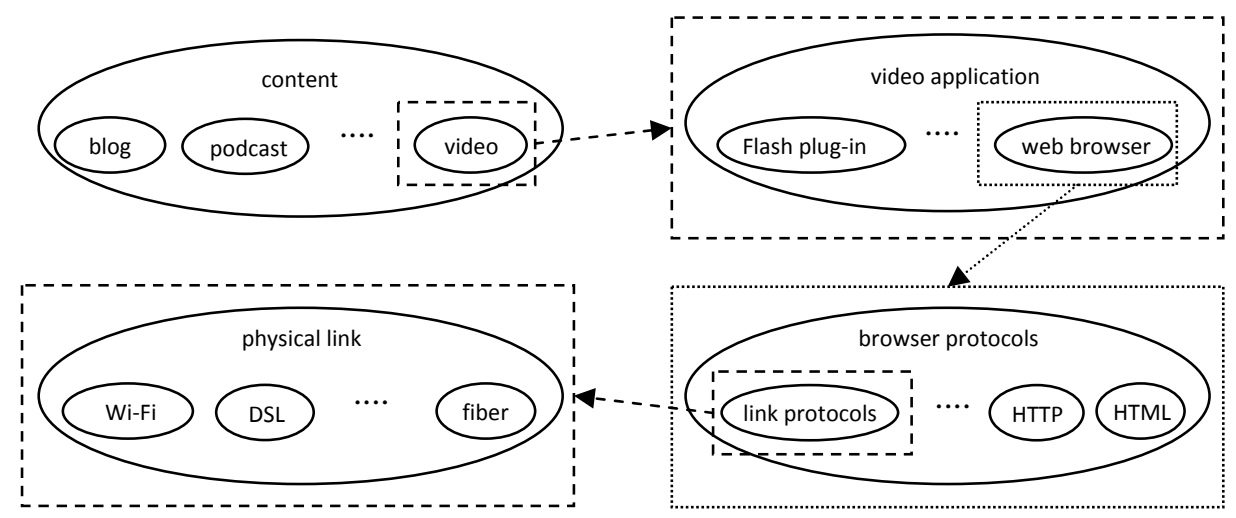

Figure 3: The Layers Model as a set of hierarchically nested systems

It is generally desirable for a system to be resilient, that is, for it to absorb substantial changes in its environment without catastrophic change. Resilient systems have good connectivity, but not too much; over-connecting leads to fragility. If a stand of trees can burn without the conflagration spreading across the forest, the overall landscape can sustain local calamities. A diversity of system elements also increases resilience by increasing the number of ways a system can resist, and recover from, a shock. Thomas Homer-Dixon (2006) points out that resilience is a public good and tends to be underprovided because no individual competitor is willing to carry the buffer needed for robustness in the face of catastrophe. It therefore becomes the responsibility of policy makers.

The consideration of nesting and cross-linking therefore support the use of principles that will be taken up in more detail in Section VI: the need for diversity in structure and constituents of a system, and the importance of weak coupling between system layers, referred to as “delegation” below.

\section{E. Novelty and Surprise}

It is very difficult to discern cause and effect in most complex systems. The interlocking interactions of sub-systems generate behavior that usually cannot be tied back to the isolated behavior of single components. In cases where cause and effect can be linked, the distance between the events (in time or space) can be very large, making the chain of causality quite tenuous. System responses to perturbations, including restoration efforts in ecosystems or interventions in markets, can be highly nonlinear and lead to management surprises.

Even systems that have been developed to have deterministic behaviors, such as biological organisms and human-engineered machines, are unexpectedly and catastrophically fragile in some rare configurations (Carlson \& Doyle 2002). Such systems are highly structured, non-generic, and have self-dissimilar internal configurations at different scales and levels of abstraction; they are not the sand piles and flocks of dumb automata so often treated in complexity theory. Their external 
behavior is typically robust, but there is a risk of rare but potentially catastrophic cascading failures initiated by quite small perturbations. Carlson \& Doyle argue that there is a trade-off between internal simplicity and robustness. Simple systems cannot operate in highly fluctuating environments; robust systems necessarily have to be complex. However, such systems can be catastrophically disabled by cascading failures initiated by tiny perturbations. For example, organisms work well under most conditions, but a single rogue mutation can trigger a fatal cancer.

Doyle \& Csete (2007) give the internet as an example of robust-yet-fragile behavior. The use of TCP/IP allows plug-and-play between modules that use the same protocols, and TCP can run transparently on any hardware that supports IP. Complexity and fragility are hidden because the protocols allow robustness to outright failures; modules can come and go. However, a small protocol error can cause catastrophic problems. Anderson et al. (2003) point out that the proven resilience of the internet does not apply to all failure modes. They note that systems obeying the syntax of a protocol may in fact be behaving incorrectly, and remark that such failures occur with surprising regularity.

Further, humans have an innate tendency to overestimate their ability to predict key trends and discontinuities (Taleb 2001, Lempert et al. 2002). Surprise stems from several sources (Lempert et al. 2002): extrapolating the present even though discontinuous jumps are common shapers of the future; under- or over-estimating the impact of an anticipated event; failing to anticipate the timing of events; differences between our revealed ability to respond to events versus what was anticipated; over-estimating one's confidence in knowing the future; and self-limiting prophesies where predictions elicit responses that counter their expectation. Human intuition is particularly prone to break down under conditions of complexity.

Human intuition is a frail guide to action when dealing with adaptive complex systems. The large number of variables exceeds our cognitive capacity, and the linear models our brains tend to prefer do not fully capture the reality of non-linear interactions. Long delays between causes and effects confound our ability to understand dynamics. Complex adaptive systems typically have both slow and fast variables; people respond best to fast variables. Changes in slow variables may not be recognized because they are imperceptible on human time scales, or because they do not fit into the mental models of observers; yet, they can often tip a system into a new state. Even when slow variables are recognized, the fact that collective action is needed to address them constrains responses. This leads to emphasis on short-term welfare that is counter-productive in the long run (Walker \& Abel 2001).

In sum, one cannot predict the outcomes of interventions in a complex adaptive system with much if any accuracy. This is true even for relatively constrained policy domains, since policy interventions almost by definition stress the system in unanticipated directions. The upshot is that "rational policy making" is little more than inspired bets rationalized both at the time of policy creation and after the fact with "Just So" stories. This is demonstrated by unintended consequences and self-fulfilling prophecies, the two faithful companions of decision making.

Ecology is rife with stories of unintended side effects. For example, southwestern states in the US are now struggling to eradicate buffelgrass from the Sonoran Desert. It was introduced by government officials after the Dust Bowl in an attempt to hold the soil and provide feed for cattle. It has unfortunately turned out to be an invasive weed that threatens the desert ecology, choking out native plants like the iconic saguaro cactus. ${ }^{43}$ Another example of biological control gone wrong is the introduction of the cane toad into Australia in 1935 to control sugar cane pests: it did not control

\footnotetext{
${ }^{43}$ By Faye Bowers, Faye (2007) “Goal for these desert troops? Bag the buffelgrass.” The Christian Science Monitor, May 31, 2007. Available: http://www.csmonitor.com/2007/0531/p01s01-usgn.html
} 
the insects, and the toad itself became an invasive species. ${ }^{44}$ Sometimes, probably more by luck than judgment, things work out; for example, the release of myxomatosis in 1950 was successful in controlling feral rabbits in Australia. ${ }^{45}$

John Sterman coined the term "policy resistance" to describe the tendency for interventions to be delayed, diluted or defeated by the response of the system to the intervention itself (Sterman 2000, sec. 1.1.1); one might also think of it as "policy ricochet". There are many examples in bio-system management. The US Forest Service's efforts to control wildfires show that fire suppression leads to a build-up in flammable material that leads to catastrophic blazes. ${ }^{46}$ Pesticides and herbicides stimulate the evolution of resistant pests and weeds, and suppressing a pest leads to a flourishing of its prey - to such an extent that the pest suddenly returns for the resulting feast in greater force than before.

The Telecommunications Act of 1996 is an example of unintended consequences in communication policy. It was intended to increase competition, but appears to have had the opposite effect. ${ }^{47}$ Another example might be government mandates of reciprocal compensation payments between carriers that led to litigation. In 1996, the FCC required all carriers to pay for call termination. Competitive local exchange carriers (CLECs) signed-up internet service providers as customers, and net payments went to CLECs rather than to the incumbent carriers, as was intended.

The novelty and surprise of complex adaptive systems suggest that policies need to be flexible to respond to unexpected developments. Policy makers should also take a holistic view (cf. the Big Picture principle) of the problem to minimize side effects. ${ }^{48}$ As John Sterman points out:

There are no side effects—only effects. Those we thought of in advance, the ones we like, we call the main, or intended, effects, and take credit for them. The ones we didn't anticipate, the ones that came around and bit us in the rearthose are the 'side effects'.

Conceiving of the internet/web as a complex social system rather than a deterministic economic or technical problem changes how one thinks about entrepreneurs, researchers, consumers, and policy makers. They are no longer external controllers of, or parameters in, a techno-economic system; rather, they are adaptive agents in an integrated social-economic-technical system. The surprise and novelty of such a system further bolsters the importance of management principles such as flexibility, since the behavior cannot be predicted, and taking a big picture view, since innovation can come from any, and across many, scales.

Table 2 summarizes the main links between the themes discussed above, and the principles that will be defined shortly.

\footnotetext{
${ }^{44}$ Blatchford, John (2007) “Cane Beetles” Apr 16, 2007. Available: http://insects.suite101.com/article.cfm/cane_beetles

${ }^{45}$ Australian Government, Department of the Environment, Water, Heritage and the Arts (not dated) "Feral animals in Australia”. Available: http://www.environment.gov.au/biodiversity/invasive/ferals/

${ }^{46}$ Fred Pearce, "Wild about fire”, New Scientist, 11 Nov. 2000

${ }^{47}$ Economides (1998) concludes that "more than two years after the passage of the Act, there is very little entry and competition in local exchange markets." Hazlett (2000) finds that "[i]mprovements in competitiveness are modest by some standards but impressive when judged against the results of other legislation with the announced goal of increasing market rivalry." He also notes that suggest that the wave of mergers was probably an unanticipated result of the Act.

${ }^{48}$ John D. Sterman (2002:505): “There are no side effects—only effects. Those we thought of in advance, the ones we like, we call the main, or intended, effects, and take credit for them. The ones we didn't anticipate, the ones that came around and bit us in the rear-those are the 'side effects'."
} 
Table 2: Complex system themes and suggested principles

\begin{tabular}{lcccc}
\hline & $\begin{array}{c}\text { Incomplete } \\
\text { Knowledge }\end{array}$ & $\begin{array}{c}\text { Cycles and } \\
\text { Transitions }\end{array}$ & $\begin{array}{c}\text { Hierarchy and } \\
\text { cross-linking }\end{array}$ & $\begin{array}{c}\text { Novelty and } \\
\text { Surprise }\end{array}$ \\
\hline Flexibility & $\mathrm{X}$ & & $\mathrm{X}$ & $\mathrm{X}$ \\
Delegation & & $\mathrm{X}$ & & $\mathrm{X}$ \\
Big Picture & $\mathrm{X}$ & $\mathrm{X}$ & $\mathrm{X}$ & \\
Diversity & & &
\end{tabular}

\section{THE FOUR PRINCIPLES}

The analogy of the internet/web to a forest ecosystem, or more fundamentally to a managed complex adaptive system, suggests that policy principles derived from these disciplines could be relevant to communication policy. This section defines and discusses four "ecosystem management principles" that provide a way to balance the competing pressures of innovation and public interest mandates, and the need for both productivity and resilience.

Supervising the internet/web, or any complex adaptive system, entails dealing with incomplete knowledge, cross-linked hierarchies, phase transitions, and surprise. Since communications systems are constantly changing, a policy approach should be built on a few simple and enduring principles that apply no matter which phase of the adaptive cycle the internet/web, and its successors, finds itself in.

The principles, summarized in Table 3 and discussed in more detail shortly, form a bridge from any given set of circumstances to an agenda of policy actions and inactions. The principles are intended to be general enough to remain relevant even as technology and business models evolve, but specific enough to be a guide to action.

Table 3: Summary of the ecosystem management principles

\begin{tabular}{ll}
\hline & Determine ends, not means. \\
Describe and justify the outcomes sought, not the methods to be used to achieve \\
them. When prescribing rules, prefer ex post to ex ante regulation. Use technology- \\
and business-model neutral rules. Give new entrants glide paths to meet policy \\
objectives. Regularly review the need for ongoing regulation, eg, by sun-setting \\
regulations.
\end{tabular}


The four principles help policy makers meet the five policy imperatives described in Section II. While every principle addresses each of the imperatives in its own way, Flexibility and Delegation are particularly suited to fostering innovation, while Big Picture and Diversity help to build resilience into the system.

\section{A. Flexibility}

Surprise, sometimes experienced as failure, is usual in complex systems; long-term prediction is impossible. Since the outcome of system evolution is so uncertain, it is unwise to pick, predict or optimize for a specific preferred outcome; at best this will lead to a mixed result, and at worst to many undesirable, unintended consequences. Ignorance of the details of how a rapidly evolving system works, combined with the likelihood of unwanted and unexpected side effects, means that regulation should fix as few parameters as possible to achieve its goal.

Regulators seldom if ever have sufficient knowledge and control of a system to be able to drive it towards a specific outcome. There are many reasons for this. Any chosen path towards an outcome is made obsolete as participants adapt to being regulated; the system changes faster than the political process that regulates it. The problem that regulation is intended to solve may be misidentified due to the complexity of the situation. Even if correctly identified, the problem may fix itself without intervention. Finally, any regulation will immediately have unintended consequences beyond just those required to address the problem at hand. All this demands regulatory humility: policies should be flexible enough to adapt to developments, becoming stronger or weaker as the situation requires.

Policy should also be flexible enough to remain viable as an industry moves through the phases of its development cycle. Any policy framework should recognize that there are times to encourage innovation, and times to allow mature incumbents to deliver the benefits of scale. It should provide a basis for adjusting the balance of intervention and forbearance at different times, and in different parts of the communication system. There have been a number of cycles in communications technology: the rise of the telegraph, then broadcasting, then the internet. ${ }^{49}$ Regulators do not have the luxury of having two policy regimes - one for stability and another for change - since different parts of the systems may be in different phases at a given moment. The diversity may be geographical (internet access is mature in the US, but booming in Asia), between industries (messaging services are mature, but online content is still changing rapidly), or in abstraction hierarchy (in the network stack, data transport is mature while social networking applications are still evolving).

Policy planning should include developing contingency plans for adverse events. Precautions should include ensuring that a regulator has the power to act in unforeseen scenarios. The bigger the downside risk, the less one should optimize for a particular expected case. For example, since imposing telecom tariff rules on all voice communication could stifle, say, in-game voice chat, regulators should hesitate about taking this step.

\section{Ex post vs. ex ante}

Neutral, open-ended policies are more likely to cope effectively with changing situations than detailed rules. However, policy makers should be ready and able to act in case something goes wrong, eg, by having the authority to impose regulations rapidly.

\footnotetext{
${ }^{49}$ Carlota Perez's analysis (2002) of 40-year technology innovation cycles provides a longer-scale context in which financing interacts with technology to generate periodic booms and busts.
} 
One mechanism to achieve this is a preference for regulation after the fact (ie, ex post rather than ex ante) since this allows policy makers to respond to problems that actually arise, rather than committing them to hypothetical scenarios. The more specific a regulation, the longer it takes to change, since it enshrines scores of hard-fought trade-offs. Regulation before the fact may, however, be necessary in cases where there is a clear and present danger of the entrenchment of market players who already have significant market power. Action may be needed to maintain the system diversity that is required for robustness.

A related method is to delegate detailed oversight, such as the conversion of principles to rules, to a regulatory agency. This allows legislation, which is slower to change than regulation, to avoid defining and setting control parameters.

\section{Means vs. ends}

It is important to separate outcomes from implementations. Policy makers have limited control over the internet/web, and can only affect its output through indirect means; even if they had the technical skill to define implementations, these would undoubtedly be a limited, and probably inadequate, sub-set of what the internet/web could come up with on its own. Regulators should therefore focus on ends, not means.

Policy makers should strive to be agnostic about technology and business models. Firms and consumers that actually generate public goods are best placed to do the detailed implementation within frameworks created by policy. The government cannot, and should not, design products. If intervention is necessary, regulation should set performance targets, not specifications. Since the outcome of interventions in complex adaptive systems like the internet/web are hard to predict, there should be a bias against detailed policy prescriptions that purport to achieve a specific outcome, eg, achieving network neutrality or requiring universal support for the TTY system to support use by deaf people.

\section{Clear boundaries}

A call for flexibility may be taken, wrongly, as a license for ambiguity. On the contrary: clear statements of desiderata and incentives by policy makers will motivate private sector action and yield better results.

Since the passage of time and the evolution of markets invariably invalidate the premises of regulation, it is useful to build in review checkpoints and termination dates, or "sunsets". Most regulation should sunset at a fixed date unless there is a proven need to the contrary; if it has to be open-ended, there should be built-in opportunities to make the case for its demise. The more detailed a regulation, the more likely it is to become obsolete in the face of social, technological and commercial innovation; consequently, the more detailed the rules, the more rapidly regulations should expire (or be revisited). For example, policies about the definition of prohibited speech might change only on the scale of decades; taxes and requirements on specific technologies, eg, an implementation of text access for the deaf, might be obsolete in 5-10 years.

Novel solutions will appear if the regulator clearly specifies the social goods that need to be delivered, and provides incentives. Transposing rules invented for another time and industry onto newcomers is likely to suppress diversity, and socially useful products might never appear. However, it must be made very clear to aspirants that they will have to satisfy all applicable policy imperatives eventually. These requirements can be met by combining clearly stated expectations with a "glide slope", that is, a time period over which new services are expected to meet these expectations. A glide slope that allows new entrants time to conform to expectations for delivering social goods will lead to more diversity, better solutions, and a more resilient system. 


\section{Examples}

Regulators have successfully used a hands-off approach in spectrum allocation, in both unlicensed and flexible-use licensed cases. For example, unlicensed allocations set a few generic limits on device characteristics, such as maximum transmission power, and prohibit harmful interference to licensed services that may share a spectrum band. Device manufacturers and service providers are free to innovate in both technologies and services within these constraints. In the 2.4 GHz ISM band, for example, one finds a variety of technologies (eg, a plethora of Wi-Fi standards, Bluetooth devices, and cordless telephones) and business models (eg, campus networks, free local hotspots, for-fee hotspot networks, and community networks). Flexible-use licensed spectrum, which gives licensees broad discretion in their use of their assignment, has also allowed dramatic innovation, such as the conversion of analog to digital networks during the 1990's. These policies have gained momentum as US policy makers and scholars across a broad spectrum have concluded that "command and control" policies that manage the uses and users of spectrum in fine detail have failed to use resources efficiently (see eg Snider 2007, Hazlett 2008).

There was a flurry of concern about interoperability between instant messaging (IM) systems in the US around 2001. Microsoft and Yahoo! pushed for IM interoperability as a condition of the America OnLine/Time Warner (AOL/TW) merger. They hoped to pressure AOL to make its thendominant IM system interoperable with competitors - such as themselves. In August 2003 the FCC removed the requirement, imposed in early 2001 during the merger review, that AOL provide IM interoperability. ${ }^{50}$ Eventually, in 2005 Microsoft and Yahoo announced that their systems would interoperate with each other; at that point, their market share was comparable to AOL's. ${ }^{51}$ The shift in attention from IM to other communication modes like social network sites, as well as third party tools that allow users to connect across all the major IM services, ${ }^{52}$ have rendered the debate moot. In the end, flexibility-by-inaction proved an effective regulatory strategy.

\section{B. Delegation}

One of the broad goals of policy is productivity, in both economic and cultural goods. Just as a forest's plants and animals are infinitely smarter about making and running a forest than foresters, the participants that make up the internet/web know more about making it work than regulators. Policy makers should therefore give them free rein. Allowing participants to experiment with new technical and commercial arrangements may obviate regulation, yielding the desired results without limiting innovation or generating unwanted consequences.

Complex systems are problem-solving machines. They show perpetual novelty, with the components and their interactions constantly adapting to whatever situation they face. For example, farmers are constantly fighting weeds that mimic crops; some crops, like rye and oats, in fact evolved out of weeds competing with wheat. ${ }^{53}$ Techno-social systems like the internet/web have the gift possessed by the heroes of Horace Walpole's fairy tale The Three Princes of Serendip (1754): the ability to make useful discoveries by accident, known as "serendipity".

Decentralized searches for consumer benefit are more efficient at finding solutions than central planning; hence, policy makers should rely on firms and civil society first, and regulate only if that

\footnotetext{
${ }^{50}$ http://www.techlawjournal.com/topstories/2003/20030820.asp

${ }^{51}$ According to Radicati Group, quoted in the Register, at that time Microsoft and Yahoo! held $44 \%$ of the worldwide IM user base between them, with AOL taking $56 \%$; see http://www.theregister.co.uk/2005/10/12/microsoft_yahoo_im_interop/

52 http://www.serverwatch.com/news/article.php/3641361

53 http://en.wikipedia.org/wiki/Vavilovian mimicry
} 
fails. Regulators can make consumer action more effective, however, if they require firms to divulge more information in a digestible and meaningful form.

Both foresters and policy makers can exert only limited control over their charges because their time, knowledge, and resources are limited. This limitation is exacerbated by difficulties in tracing cause and effect, which severely complicate diagnosis, prognosis and prescribing remedies.

Control of a system can be achieved only if the repertoire of the controller is at least as great as the variety of the situation to be controlled. ${ }^{54}$ Further, the weaker and more uncertain the regulatory capability, the more hierarchy is needed in the organization of regulation and control to get the same result. ${ }^{55}$ These systems laws imply that a regulator cannot control a system directly; it needs to work through intermediaries. This accounts for the control hierarchy in communications, as where the US Congress enacts general laws and the FCC implements regulations at the federal level, with a similar structure (legislatures and public utility commissions) at the state and local levels. Legislation and regulation at the higher level necessarily has to be general and focus on just a few parameters. There isn't enough variety or responsiveness in the repertoire of, say, the FCC to match the fluid situation in the marketplace. Agents closer to the action are more able to exert effective control over their part of the system, and generate change.

Even if it were possible, close management is often harmful. Managing target variables in natural systems leads to slow changes in other ecological, social and cultural components that can ultimately lead to the collapse of the entire system (Holling et al. 2001, Ch. 1). For example, effective flood control leads to higher human settlement densities in the fertile valleys, and a large investment in vulnerable infrastructure. When a large flood eventually overwhelms the dams and dikes, the result is often a dramatic reconfiguration of the social and economic landscape along the river. Ultimately, technocratic management of single parameters leads to instability of the entire system.

In some cases, there may not be an economic rationale for market participants to address a social problem without incentives and threats. This can occur in the provision of access to the disabled, for example. A market may also be too fragmented or fractious to come to solution that has the necessary economies of scale; this was arguably the case with the conversion to digital cellular service in Europe. Regulators may still, however, be able to achieve the desired outcome without having to act simply by taking powers and/or threatening action. An exception can be made to this approach if there is a clear prospect of loss in consumer welfare through lock-in by an unregulated incumbent.

Overall, regulators can guide outcomes in a positive direction by providing clear statements of what is required; setting up appropriate incentives and deterrents, without specifying implementations; giving individuals, civil society and the market time to meet the challenge; and finally intervening with detailed rules if voluntary action has failed.

\section{Examples}

Peer-to-peer (P2P) file distribution started as a hacker tool often used to circumvent copyright, but is now increasingly being used by commercial firms. NBC announced in September 2007 that it would use P2P delivery to enable viewers to watch TV shows as ad-supported downloads. ${ }^{56}$

Examples in telecoms where managing a single parameter led to instability include the regulation of international call settlement rates. High government-protected rates for call termination resulted in competitive carriers looking to bypass the incumbent carriers to terminate calls in a country. This

\footnotetext{
${ }^{54}$ Ashby’s "law of requisite variety”, see Skyttner 2001:92

${ }^{55}$ Aulin and Ahmavaara's "law of requisite hierarchy”, see Skyttner 2001:92

${ }^{56}$ http://blog.streamingmedia.com/the business of online vi/2007/11/nbc-direct-to-u.html
} 
decreased incumbent revenue and destabilized their business model, and eroded the cross subsidy of local by international calls.

The video game industry set up the Entertainment Software Rating Board (ESRB) in 1994 to assign computer and video game content ratings. The threat of Congressional action undoubtedly concentrated the minds of the industry. ${ }^{57}$ Policy makers got the outcome they desired, without having to get into the details of defining ratings themselves.

Another successful instance of delegation with a threat of action was the approach of the US Government (USG) to internet governance: it delegated authority to the IETF, a technical body staffed by independent technical experts whom the USG trusted to make the "right" (technology-led, market-biased) decisions without direct supervision. The alternative governance forum, the International Telecommunications Union, was controlled by dirigiste governments and their preferred suppliers, and the USG considered the IETF as the lesser of two evils even though it entailed substantial delegation of power (Cowhey, Aronson and Richards, in manuscript).

\section{C. Big Picture}

Systems have overall properties that cannot be predicted by an analysis of their sub-systems. Further, one cannot optimize the system piecewise: if each sub-system is operating at its best, then the system as a whole will not be at maximum efficiency (Skyttner 2001:92). Piecewise regulation ignores these emergent properties of a system, and will lead to sub-optimal results.

Consequently, policy makers should take a holistic view of the potential sources and kinds of solutions to policy problems. Casting a wide net for solutions to problems is most likely to find good answers. Generic regulation is more likely to foster productive innovation than narrow sector-, technology-, or business-specific rules. For example, existing instant-messaging tools offer solutions to web communications for the deaf; text-via-telephony solutions needn't necessarily be mandated. A holistic approach also implies a broad view of the regulator's role: not only to regulate, but also sometimes to forbear.

Parts of the US communication industry that are regulated by different titles of the Communications Act. These silos have the advantage of being a sub-set of the entire system, and are thus easier to characterize and control; for example, life is simpler if one can treat broadband infrastructure and content rules separately. However, broadband speeds and limitations influence the kind of content that emerges, and content drives demand for infrastructure. One therefore needs a "no silos" approach.

One might take "don't regulate in silos" to mean "don't regulate by classification". This is probably impossible; regulators instinctively, and rationally, try to categorize new problems. Once a regulator can fit a new service into a category, regulatory action follows automatically; one does not have to go back to first principles in every case. However, in a dynamic world such as the internet/web, categories are constantly shifting and changing. Categorization has little lasting value. A principled approach, as advocated here, is better able to maintain its value over time.

The details of a "no silos" approach depend on what counts as a silo. It is now generally accepted that internet access services should be regulated regardless of the transport medium used. Since different industries used to be defined by their transports (wire-line telephony, broadcast TV, cable $\mathrm{TV}$, cellular telephony, etc.), this implies that regulation should cross industry silos defined by transport medium. The unit of regulatory analysis is now the service, defined as a user experience. Similar services offered by different industries would be considered as a group in a holistic

\footnotetext{
${ }^{57}$ Nichols, Peter M. "Should video game makers police themselves? The issue sparks a dispute between the industry's titans, and a chance of legislation from Congress.” New York Times, 17 Dec. 1993
} 
approach; taken together they may entail sufficient diversity and choice to obviate close regulatory supervision. Regulation should be applied on a service basis, and not to components that make up a service. Similar functionality might be offered in different markets as parts of different services, eg, real-time voice communication in the telephony and on-line game markets. Telephony regulation of real-time voice would not be transposed automatically to on-line games simply because it contains a voice communication feature.

\section{Examples}

Many properties of the internet/web do not arise directly from the behavior of a particular part. For example, packet traffic volume depends on the amount of fiber capacity, transport and application technologies, the financial health and business arrangements of service providers, the shifting popularity of particular applications and sites, and legal initiatives. Each of these factors depends on the others; the resultant traffic volume is an emergent property.

A narrow focus also reduces the robustness of a managed system. While fires sometimes destroy forests, suppressing them for too long increases in leaf and branch litter can lead to catastrophic burns. $^{58}$ The soil structure and large trees around which forest communities are built survive through medium-sized fires, allowing rapid rebuilding; in big fires, the soil is sterilized and large trees are felled. Similarly, while occasional disruptions in industry revenue arrangements are uncomfortable for vocal incumbents and stressful for entrants, they prevent wrenching restructuring later on. The inflexibility and regulatory capture that results from industry-specific rules can limit competition and valuable innovation, eg by protecting rural telephone companies from wireless competition.

If there are multiple pre-existing categories for a converged service (eg, video), and regulation is deemed to be necessary at all, the regulator should start with the weakest available regulation, not the strongest; one can always ratchet up the pressure if necessary.

\section{D. Diversity}

A lack of bio-diversity was a contributing factor to several agricultural disasters, including the Irish potato famine of 1846, the European wine industry collapse in the late 1800s, and the US Southern Corn Leaf Blight epidemic of 1970. A diverse range of organisms contributes to the resilience of agricultural and wilderness ecosystems, their capacity to recover from environmental stress and their ability to evolve. It increases food security for humans, too. Species diversity reduces external input requirements in farming by increasing nutrient availability, improving water use and soil structure, and controlling pests naturally. ${ }^{59}$ Wells \& Darby (2006) argue that economic diversity can bring social benefits that parallel those to be found with bio-diversity including economic resilience, variegated opportunities in terms of employment choices, regenerating localities, and activities that are in harmony with the characteristics of the locality, both human and natural.

System diversity consists in having a large variety of different agents, with different goals and means, at many different scales in time and space. In an industry context, diversity entails nurturing new entrants (both new firms and new industries) and allowing them time to mature. Once a tree seedling planted during a wetland restoration has become established, it no longer needs protection from off-trail walkers. Similarly, early stage companies and industries may need protection from competition for a limited time during their infancy, but not afterwards.

\footnotetext{
58 “Effects of Fire Suppression”, Forest Encyclopedia Network, http://www.forestencyclopedia.net/p/p4/p142/p165/p167/p170

59 "Biological diversity in food and agriculture," Food and Agriculture Organization of the United Nations, available at: http://www.fao.org/biodiversity/index.asp
} 
Diversity in a managed system may need to be maintained. The competition that is at the root of the success of markets also provides incentives for firms to establish monopolies, duopolies or cartels. These structures, if unchecked, allow firms to extract non-cost-based rents, and extend their dominance into adjacent markets. While market dominance has benefits in terms of standardization and stability, and is arguably efficient, it reduces diversity and thus system resilience. Dominance may also reduce innovation. ${ }^{60}$ It is therefore necessary to limit the ability of firms to abuse significant market power to the detriment of the overall health of the system.

It may be argued that a reduction in diversity, such as through de facto or de jure standardization, is beneficial because it allows economies of scale and provides some stability in otherwise chaotic markets. Standardization is a regular feature of the information technology industry: HTTP won out over Hyper-G, TCP/IP overshadowed X.25, and the Windows platform became a monopoly. Reduction in diversity amounts to an efficiency/resilience trade-off; the resulting system is more efficient, but less resistant to shocks. Differences in regional technology policy can protect diversity, as happened with the regulatory standardization on GSM in Europe in contrast to the variety of cellular air interfaces in the US.

The European approach to telecommunications regulations provides a framework for encouraging diversity through market entry (Marcus 2002). It focuses on responding to market power. If a national regulator finds that a firm possesses Significant Market Power (SMP) within a defined market, it may impose obligations including transparency, non-discrimination, accounting separation, access to and use of specific network facilities, and price controls. If there is no SMP, any such obligations must be rolled back. The current review of the EU Framework Directive indicates that European regulators continue to be mindful that diversity is an important component of a healthy communications system. It proposes that regulators will focus their resources on the market sectors in which the dominance of incumbents has been least challenged. ${ }^{61}$

The approach embodied in the Ecosystem Management Principles is based on two premises: (1) that the principles embody useful lessons of complex adaptive systems theory, and the practice of ecosystem management; and (2) that complex systems theory and forestry are good models for the internet/web. The precise formulation of the principles matters less, however, than the principleoriented philosophy that underlies them. The same premises may lead others to different formulations, but a principles approach is likely to remain.

While the principles (in whatever form) may be necessary, they are not sufficient. They are designed to address the aspects of dynamic complexity of the internet/web, and not good governance in general. All the standard lessons of political science apply, and they can be framed as additional principles of good governance. The "Five Principles of Good Regulation" developed by the Better Regulation Executive of the UK Department for Business, Enterprise and Regulatory Reform exemplify general principles of governance. ${ }^{62}$ They state that any regulation should be transparent, accountable, proportionate, consistent, and targeted only at cases where action is needed. ${ }^{63}$ In a

\footnotetext{
${ }^{60}$ The link between firm size and innovation is unclear, particularly when research networks, partnerships and collaborations are taken into account. For example, Tether (1998) casts doubt on the belief that small firms are more innovative, or more efficient innovators, than large firms. Nicholas (2003) argues that strong market positions are powerful engines of technological progress, despite market power abuses.

${ }^{61}$ See eg http://ec.europa.eu/information_society/policy/ecomm/tomorrow/reform/index_en.htm, http://www.ovum.com/news/euronews.asp?id=6374

62 See http://www.berr.gov.uk/bre/

${ }^{63}$ More detail on the meaning of these terms can be found at http://archive.cabinetoffice.gov.uk/brc/upload/assets/www.brc.gov.uk/principles.pdf
} 
similar vein, ministers from OECD countries who met in Ottawa in October 1998 to discuss electronic commerce stated that "Government intervention, when required, should be proportionate, transparent, consistent and predictable, as well as technologically neutral.”,64

\section{E. The importance of Resilience}

Occasional catastrophic failures cannot be designed out of a complex adaptive system. They're a consequence of its adaptability (Carlson \& Doyle 1999). Even without such unpleasant surprises, unexpected behavior and perpetual novelty are important attributes of complex systems.

Collapse and renewal are essential to the long-term productivity and stability of ecosystems (Gunderson \& Holling 2001). In politics, the periodic replacement of political leadership flushes out corruption and provides new insights, even though a price is paid in the loss of expertise. Striving for immutability sets up the conditions for a catastrophic collapse; for example, the intensive use of lawn chemicals leads to superficial health, but force-feeds the grass while denuding the soil of organisms. That leads to feeble grass that is vulnerable to diseases and weeds. ${ }^{65}$

During the exploitation and conservation phases of an adaptive cycle, a forest or an industry is stable, and produces many apparently secure jobs (and happy voters). In industry, gains from scale lead to consolidation, and firms not competing directly with the major players benefit from standardization and the creation of a product platform. However, the situation is fragile, because the majors represent a single point of failure. ${ }^{66}$ When they do fail, damage can be limited if the sector is still growing in other areas, eg, internationally or in other parts of the value chain. Policy for the internet/web should therefore not only prepare for collapses; it should build in the conditions that allow periodic small collapses and minimize the likelihood of rare catastrophes.

A resilient system is one that can maintain its structure and function in spite of experiencing disturbances (Holling \& Gunderson 2002). In cases where there is uncertainty about outcomes usually the case for complex systems - it is better to choose robustness rather than optimality. A robust strategy is one that performs reasonably well, compared to the alternatives, over a wide range of plausible scenarios (Lempert et al. 2002). Optimality would select the strategy that performs best in the most plausible scenario, not necessarily the one that is most resilient regardless of scenario.

Resilient policies comprise strategies that perform well, compared to the alternatives, over a wide range of plausible scenarios. The various stakeholders may have different ideas about what constitutes success. Resilient policies will therefore also perform reasonably well when judged against the many value systems held by different parties to the decision (Lempert 2003, Walker \& Abel 2001).

A variety of techniques increase the resilience of policies: trying not to pick or determine a single preferred outcome; including contingency plans for the worst case; designing policies that adapt to changing circumstances by evolving over time in response to new information; modeling the systems dynamics of the problem under consideration; and analyzing the robustness of chosen strategies against many plausible futures.

\footnotetext{
${ }^{64}$ Statement available at http://www.ottawaoecdconference.org/english/announcements/e_oecdrelease.htm

65 “Greener lawn care” ColoradoSprings.com, June 27, 2007. Available: http://www.springshomeandgarden.com/fullStory.jsp?id=6019

${ }^{66}$ Cross-scale consolidation is not evident in the internet/web at the moment, but presents a threat to robustness should it occur. One scenario is that consolidating network operators vertically integrate into the content business, in the same way that cable companies used their market power to take stakes in content providers (cf. TCI growing into Liberty Media).
} 
Loose coupling between internet/web sub-systems at different hierarchical scales improves system robustness. Aspects of the layers approach can therefore be justified in terms of achieving system resilience. Government should not take action that encourages vertical integration. However, breaking up vertical integration after the fact should be approached very cautiously, since one cannot predict the consequences for the system as a whole.

There are two aspects to policy resilience: policies that lead to resilient systems, and policies that are themselves resilient to changes in external circumstances. All four principles contribute to the first aspect, and the Flexibility principle in particular addresses the second.

The policy principles help to improve resilience in various ways:

1. Diversity. A variety of participants ensures that a local collapse leads to a rapid restart of system function by facilitating entry of newcomers in times of disruption. If there is a monopoly, particularly at a variety of system scales, then a failure is likely to cause widespread disruption. ${ }^{67}$

2. Flexibility. Technology- and business model-neutral policy will stimulate diversity, which will improve resilience. Incorporating the possibility of unexpected events into regulatory frames, rather than simply optimizing for a single scenario, will lead to more robust policies.

3. Delegation. Allowing sub-systems to evolve at their own pace allows different parts of the system to be at different stages of maturity. If surrounding parts of the value chain are stable when one is disrupted, the overall system will continue to function. Clear goals focus participants on long-term outcomes, and sustain momentum through periods of creative collapse and renewal. Policy expiration dates remove unused regulations, and reduce the possibilities of unexpected interactions.

4. Big Picture. Seeking the health of the communication system on a broad scale rather than narrowly optimizing for the interests of particular incumbents allows for more flexibility and experimentation, which enhances resilience. Ensuring that different system scales are not too tightly coupled prevents disruption at one scale from causing a system-wide collapse. ${ }^{68}$

System resilience can be further improved by not operating the system in a regime that is vulnerable to shocks, even if that were the most economically efficient favoring robustness over efficiency. The bigger the downside risk, the less one should optimize for a particular expected case.

\section{THE PRINCIPLES IN ACTION}

This section illustrates how the forestry/complex systems metaphor, and the principles derived it, can be applied to policy challenges. It tackles questions such as the degree of intervention required

\footnotetext{
${ }^{67}$ Diversity, both social and ecological, is an important determinant of ecological resilience in rangelands, which are regions between deserts and agricultural zones where people make their living from pastoralism. Examples, taken from Walker \& Abel (2001), include: the juxtaposition of soils with differing abilities to accept and store rainfall enables vegetation on some soils to survive through periods of sparse rainfall and on others to grow well under conditions of higher rainfall; plant communities with high species richness with functional types (groups) of species ensure a variety of responses to different environmental disturbances; mixed grazer and browser animal populations increase forage and marketing options, reduce drought risk, and slow shrub encroachment; diverse enterprises linked to different markets and requiring different weather conditions reduce risk; a range of energy sources (human labor, horses, oxen, fossil fuels) widens resource-use opportunities; a relatively large workforce with a mix of ages and sexes expands adaptive opportunities; having access to a region with spatially variable climate enables survival through mobility; having access to diverse land systems at regional scales offers a range of opportunities in time and space.

${ }^{68}$ This is widely seen in political systems, where different parts of government are replaced at different rates. In the US, for example, federal elections are held every two years for Congress, four years for President, six years for the Senate, and federal judges have life-time appointments.
} 
in video programming on the web, the best way to ensure that the disabled can benefit from the internet/web, and providing access to emergency services in the context of internet-enabled applications.

The principles that are invoked will be marked in the right margin as they appear.

\section{A. Video Services}

Traditional video programming is heavily regulated. Policy makers' overall objectives are (as usual) productivity and resilience: there should be a large amount of high quality video content that meets consumer demand and socio-political needs; and production should continue without pause in spite of dramatic changes in technology and business models. Rules cover a wide spectrum, including broadcast technology standards, content regulations and production quotas, access for people with disabilities, limits on the types and times of advertising, and control over content such as sex, violence, and political speech.

The public interests served can be analyzed in terms of the policy mandates described in Section II above, Permanent Policy Imperatives. Government revenue considerations include hidden subsidies to local content producers through quota requirements on broadcasters, and funding mechanisms for public broadcasters. Consumer protection measures include rules to ensure that the disabled have access to content, eg, through closed captions and embedded audio descriptions. Policy makers pursue economic vitality by favoring local over foreign producers through hidden subsidies, industrial policy, and content quotas. They may also intervene if market power seems to prevent entry of new services. Many content rules are framed in terms of protecting local cultural values, either in terms of production rules, content preferences, or rules on ancillary material like advertising content. However, they can be hijacked by ambitious policy makers seeking to burnish their populist credentials in their own self-interest.

The traditional video content sector (film and broadcast television) is relatively orderly, with a limited number of participants; it looks a lot like industrial farming. The regulator tries to ensure that the "right" content crops are produced through subsidies and rules that govern a well-defined and stable group of policy and industry players.

Video content in the age of the internet is more like multiple-use national forest land with its many natural settings, uses, and constituencies. There are many ways to consume video: as broadcast content over the air, satellite, and cable; and on-demand via proprietary cable and telco networks, and the public internet. There is a spectrum of quality from amateur to professional. Viewers have widely varying needs and wants, from education and entertainment to politics and building social networks.

Simply re-applying old regulations to new services and technologies can limit the production of new goods: socially beneficial new services that don't fall in traditional categories premised on semi-monopolies and well-defined distribution channels might never appear because of onerous requirements or lack of hidden subsidies. For example, if all internet video had been required to support video description ${ }^{69}$, there might never have been the flood of user-generated on-line video material using American Sign Language. ${ }^{70}$

\footnotetext{
${ }^{69}$ Video description is an aural description of a program's key visual elements that is inserted during natural pauses in program dialogue. See eg http://www.fcc.gov/cgb/dro/pam.html; similar rules apply in many other countries.

${ }^{70}$ http://www.youtube.com/results?search query=ASL+vlog\&search=Search
} 
Internet/web content blurs existing classificatory boundaries. A piece of video can show up on a $\mathrm{TV}$, a PC, or a phone; it can be delivered as a real-time broadcast, off-line podcast, or on-demand web video. Depending on the context and interpretation, different traditional categories might apply, and different obligations would ensue. Applying the most stringent rule to all cases will squelch creativity; it would be like always using the most potent insecticide once a pest is found, no matter the effects on a particular stand of trees.

Regulators should think broadly about the implications of regulation, since the highly connected nature of the internet will propagate the effects rapidly. For example, former FCC Commissioner Susan Ness (2006) argues that an unintended consequence of the 1996 Telecommunications Act was that the prolonged Digital Television transition has delayed freeing up spectrum needed by first responders. At the other end of the political spectrum, the Electronic Freedom Foundation has concluded that the anti-circumvention provisions of the Digital Millennium Copyright Act (DMCA) have not been used as Congress envisioned; for example, rather than focusing on pirates, many copyright owners have chosen to use the DMCA to hinder their legitimate competitors, which impeded competition and innovation. ${ }^{71}$

Consequently, policy makers should devise the least constraining rules consistent with the situation at hand, and build in mechanisms to revisit them from time to time. ${ }^{72}$ For example, regulators should not lock in a particular funding regime for local video content, eg, by cloning existing rules for broadcast TV production quotas. Since internet video is still evolving rapidly, it would be wise wait for the situation to stabilize. Suitable subsidy mechanisms for desirable content using new distribution channels - if needed at all - may only become evident as the new channels mature. The web may generate content without government incentives. In January 2008, Wikipedia claimed to have more than 75,000 active contributors working on some 9,000,000 articles in more than 250 languages. ${ }^{73}$ So much local material could not have been produced commercially even at the behest of a policy mandate, and that could not have been foreseen.

Looking ahead, advertising rules tailored to commercials interspersed between segments of a show cannot be generalized to product placement; product placement rules geared to practices at a given time will be made obsolete by workarounds that industry finds.

There is always a temptation to tailor rules to a particular case. However, this leads to policy that is optimized for a particular outcome, and which will quickly become moot - a failure of resilience in the system. For example, Guo (2003) relates that in the early days of Chinese television the main responsibility for broadcasting rested with local governments, under the administrative authority of the State Council. This worked while there were fewer than 30 regional stations. The exponential growth of Chinese television in the late 1970s rendered this system obsolete and ineffective, which led to a four-tiered broadcasting system being introduced in 1982.

A healthy garden, forest or farm has a diverse mix of species; monocultures need debilitating amounts of fertilizer and pesticides, and are susceptible to sudden infestations. The analogy to the media industry is well understood in North America and Europe, where there are periodic controversies over the dangers of the consolidation of media power. However, existing diversity rules that were developed for the silo business model may not apply to new media; in fact, they may

\footnotetext{
71 “Unintended Consequences: Seven Years under the DMCA”, Version 4, April, 2006. Available: http://www.eff.org/wp/unintended-consequences-seven-years-under-dmca

${ }^{72}$ The end of regulatory supervision inevitably generates a great deal of angst, and may generate new rules; see eg “Cable Regulation End Concerns Kennard,” Television Digest with Consumer Electronics, 5 Apr. 1999 at http://findarticles.com/p/articles/mi_m3169/is_14_39/ai_54301407/pg_1 about the end of cable rate regulation in 1999.

${ }^{73}$ http://en.wikipedia.org/wiki/Wikipedia:About
} 
actively discourage new kinds of content that competes across traditional boundaries, and is funded in new ways. ${ }^{74}$ This will benefit incumbents, and while it might protect the industry from a painful restructuring for a while, it will limit the production of content and postpone the inevitable restructuring. For example, a panel of economists convened by the AEI-Brookings Center argued in 2006 that forcing new providers of video services to obtain franchises in every municipality, which is intended to protect local consumers, would discourage competitive entry. ${ }^{75}$ Looking ahead, rules that encourage entry by forbearing from imposing public broadcaster rules on start-ups will generate innovation, services, and revenues. Limiting the ways in which advertising can be used to fund programming, particularly for emerging services, can reduce the diversity and vitality of content providers by putting a cap on revenue potential.

In summary, policy makers should focus their efforts on defining what's acceptable and what's not, and providing incentives for desirable outcomes. They should reserve powers to penalize providers that eventually don't meet their criteria, rather than trying to specify in detail, in advance, how content should be produced and distributed.

\section{B. Real-time voice services}

Internet voice conversations pose a significant challenge to policy making since many aspects of these applications overlap with a substantial body of traditional voice telephony ("Plain Old Telephony Service" aka POTS) regulation. These rules emerged in the context of monopoly service providers, and it has proven difficult to disentangle provisions that address antitrust concerns, meet other social needs as a condition of monopoly (eg, cross subsidies, universal service), and speak to social interests unrelated to market structure (eg, public safety). POTS rules are also anchored in the world of regulatory silos, since they were developed at a time when only a particular industry using a specific technology infrastructure - wire-line telephony - could deliver voice conversations.

The policy imperatives of Section II are all in play. Revenue is a central motive for regulation. Regulators need to continue to generate tax income for existing programs such as universal service even as the revenue base in traditional industries shrinks. There are calls to spread the tax base to new services (eg, cellular telephony and internet voice communications), since the old ones (eg, wire-line long-distance) are dying. Universal service exemplifies another policy imperative: the consumer protection interest of providing access to various disadvantaged populations when markets would fail to do so. Public safety may require the disabled to be able to use to emergency services; this is a high profile subset of the larger consumer protection question. Fees are often presented as a way to raise funds for emergency services, thus invoking the public safety imperative. The "sixth imperative” of self-interest is visible when policy makers seek to score political points by supporting first responders, or when they advantage incumbents with an eye to future sinecures.

The voice communications business is like a forest where a lot of big trees have been blown down after being weakened by age and shifting climate. The reassuring old food web in which every organism had its place has gone; in its place is a buzzing confusion with many new species contending for their share of sunlight, and the promise of a new community better suited to new circumstances.

\footnotetext{
${ }^{74} \mathrm{Cf}$. the debate over whether satellite radio in the US is a monopolizable market or part of a larger music industry http://en.wikipedia.org/wiki/Sirius_and_XM_merger, and the perennial controversy over US media ownership rules, see Stephen Labaton, "Plan Would Ease Limits on Media Owners," The New York Times, 18 Oct. 2007 at: http://www.nytimes.com/2007/10/18/business/media/18broadcast.html?pagewanted=all

75 “Economists' Statement on U.S. Broadband Policy,” AEI-Brookings Joint Center for Regulatory Studies, Related Publication 06-06, March 2006. Available: http://www.aei-brookings.org/admin/authorpdfs/page.php?id=1252
}

Delegation, Flexibility 
There are many open questions in this regulatory area. I will consider fees, access to emergency services, and access for the disabled.

1. Fees

There is nigh-impenetrable thicket of telecom fees and taxes at local, state and federal level. As traditional business is supplanted by IP-based industries, however, this revenue base shrinks. To use a forestry metaphor, the old growth is dying, but the new forest is not yet established. ${ }^{76}$

In the US, the inventory of taxes and fees include: federal and state Universal Service Fund levies, including subscriber line charges, designed to serve poor/rural users but also to keep regional telephone companies afloat; state and local 911 levies, usually a fixed, per-user monthly fee, to help local first-responders; fixed, per-user monthly federal and state Telecommunications Relay Services fees, to fund service to help the hard of hearing; and local access charges, to subsidize local telephone companies.

The protection of these revenues streams through the blunt transposition of monopoly-inspired 20th century "revenue requirements" could crush emerging consumer-friendly services. New suppliers of voice communication are still experimenting with business models and have not yet reached equilibrium; for example, ad-supported or loss-leader offerings may supersede traditional subscription services, but generate less (or no) revenues to tax. Voice traffic is dwarfed by the volume of video packets on the internet. Its marginal cost is essential zero, and over the long run - in the absence of regulatory intervention - the price would tend to zero, too. Fee/tax policies pose a real risk of preventing that from happening.

Imposing old taxation models on the internet/web could foreclose the emergence of such services, since they would have to charge for otherwise free services in order to pay \$5-10 per user per month to satisfy the revenue needs of local, state and federal programs premised on traditional telephony taxes.

The revenue imperative cannot be evaded, however. Legislators will not tolerate the bypass and arbitrage opportunities afforded by internet voice for long unless there are alternative revenue streams. The default answer -subjecting new-era "carriers" to the last-century fee structure, as already seen in the creation of the "interconnected VoIP" regulatory category - is seductive but counter-productive because it forestalls the growth of new industries that may be revenue sources in the long run.

Part of the answer is to reduce revenue needs. For the rest, the Ecosystem Management Principles suggest taking a holistic approach: rather than trying to tax individual services and technologies, which keep changing, revenue should be generated from as broad a base as possible. ${ }^{77}$ In a perfect world, telecom-specific taxes would end - but this is politically impractical. The best that can be achieved is probably a broad-based scheme that covers communication services in a technology- and business model-agnostic way. For example, one could levy per-packet taxes on the data stream that a user consumes, regardless of the use to which it is put. A per-packet tax would be progressive in that heavy users would pay more. A less equitable but cheaper-to-administer alternative would be to levy per-connection or per-household internet access fees.

\footnotetext{
${ }^{76}$ Three quarters of the merchantable pine in the central/southern interior of British Columbia is at risk of disappearing by 2015 due to the ravages of the mountain pine beetle. This is due to a confluence of factors, including increasingly warm winters since the mid-90s that have allowed the pest to prosper, and a significant increase in mature stands of lodge pole pine due to a deliberate policy of fire suppression. See eg Sharon Oosthoek, "Nature 2.0: Redefining conservation”, New Scientist 7 Jul. 2008, at http://environment.newscientist.com/channel/earth/mg19926631.400-nature-20-redefining-conservation.html

${ }^{77}$ It is a truism in economics that taxes are most efficiently levied over the broadest range of incomes.
} 
It is important for policy makers to declare the objective that all services should eventually generate a share of revenues, but to leave the mechanism open to experimentation.

All similar services should bear the same burden. However, there is a countervailing consideration: taxing newcomers like PC-to-PC voice features can prevent new entrants growing to the scale where they can compete with incumbents. When a forester tries to encourage re-growth with diverse native species, he may need to protect the seedlings from being eaten by local wildlife. The goal is to have the same expectations on all when they are able to meet them, but not to impose the old mechanisms on the new entrants.

\section{Access for people with disabilities}

Providing the disabled with access to communications services can require government intervention since the market for such specialized services may not be large enough to motivate firms to provide such features. However, state involvement needs to be more agile than before, since the communications environment is much more complex and rapidly-changing.

Regulation should provide clarity on the requirements that society has for supporting disabled citizens, but leave it up to industry and civil society to find solutions. Allowing differential pricing is one way to make it more commercially attractive, but is unlikely to be sufficient.

A regulator should have an open mind about how mandates are to be met; existing commercial offerings intended for the able-bodied may be sufficient. For example, IM might be sufficient to carry text alongside audio for the deaf, without mandating Telecommunications Device for the Deaf (TDD) technology for web services.

Clear goals and support for voluntary action should be complemented by incentives/penalties as well as long enough lead times to allow manufacturers to get products into the pipeline. Mandated disclosure of product accessibility can help manufacturers market their products as well as assist the disabled in finding the best solution. Struggles over providing support for the hearing-impaired on the web provide a good argument that there should be flexibility in rules over time.

Legacy feature sets and requirements such as TDD should not be imposed unthinkingly on new services. Locking in TDD technology helps only a small number of legacy manufacturers, to the potential detriment of a new generation of users who could benefit from innovation. Premature regulation will stifle the invention of better-than-traditional access solutions on new platforms. Users who depend on TDD should be helped to transition to new technologies; it is more productive to convert the small legacy base of TDD equipment to new technologies than to shoehorn new technologies into a Sixties mold. A sunset on TDD technology can be tied to meeting needs for emerging services; this provides an incentive for firms to meet their social obligations.

\section{Access to emergency services}

Telephones have provided a single point of access to emergency services ("911" for short, using US terminology) for many decades. ${ }^{78}$ As new live communication services such as VoIP and text messaging emerge, the question arises: what emergency access mandates, if any, should they bear? A park system metaphor might be that new forests solve problems in new ways: an old niche might

\footnotetext{
78 The Wall Street Journal reported AT\&T's adoption of "911" as a nationwide emergency telephone number in on January 12 and January 15, 1968; see http://www.911dispatch.com/911/history/wsj_15.html. It is striking that questions of proper routing of calls, a topic with VOIP services today, was also an issue at that time. In the United Kingdom, the "Whitehall 1212" was introduced in London in 1934, and “999” in July 1937; see http://www.fire.org.uk/advice/999history.htm. European Union countries are gradually introducing a common emergency code, “112”, since 1991; see http://en.wikipedia.org/wiki/Emergency telephone number
} 
house a new species, or a completely different niche structure might arise that distributes resources in new ways.

Consumer expectations regarding 911 access using these new media are still in flux. They may or may not deem it necessary that an application that includes a real-time voice communications feature, such as an on-line game, or a non-voice telephony service like SMS, should offer emergency services access; and this judgment is likely to change over time.

Incumbents can use emergency service mandates, such as interconnection to public safety access points, as competitive weapon against new entrants. The funding of emergency service infrastructure such as public safety access points is based on the structure of traditional telephony; imposing fees and interconnection requirements on new service entrants creates a significant burden of entry.

New entrants should be allowed a grace period to arrive at the level of service offered by longstanding competitors, and to develop appropriate solutions. The imposition of legacy mechanisms gives incumbents a way to limit market entry, to the detriment of the long-term productivity and resilience of the market. New forest communities have new ways of doing things; species arriving from outside fill old niches, and create new ones. Regulators should not stifle new services by forcing them to conform to old rules; a new service may not need a 911 mandate, even though it contains voice conversation as a feature. If users eventually come to expect it, then apply mandates on providers only if they have not provided it. It is reasonable to expect that services that become pervasive and subject to consumer expectations should provide access. However, the service may not look like a traditional 911 call. Regulators should assert that emergency access service will be expected once a service reaches a clearly defined critical mass - eg, that $30 \%$ of the population uses the service at least once a week - but then leave it to firms to develop solutions. During the transitional period, new services should be given the opportunity to decline to offer emergency access, provided that this lack is clearly disclosed.

Providing 911 access on new internet/web services reveals an apparent contradiction in applying the forestry/complex systems metaphor. On the one hand, thinking holistically about the system suggests that all providers of communications services should be treated equally, and that everybody, new entrants included, should provide access. On the other hand, fostering creativity through allowing experimentation and increasing robustness by encouraging new entrants implies that newcomers should be given time to meet access requirements; different rules should apply to old and new.

The paradox is resolved by requiring new services to provide access, but not mandating that they use traditional methods. The requirement to provide service is also graduated, allowing time for entrants to arrive at the level of service offered by long-standing competitors. Not imposing the full burden immediately allows novices to develop their competence; it may turn out that the service fails for other reasons, and one will not be left with legacy rules that will deter future entrants. The PSTN interconnect condition on emergency access mandates for VoIP services is a politically expedient proxy for a glide path; it presumes that VoIP services that connect with the PSTN are sufficiently mature to be equivalent to traditional telephony services.

\section{REFINEMENTS AND GENERALIZATIONS}

\section{A. Two Useful Tools}

Policy principles provide a guide to action, but do not specify in detail what a policy maker should do; for that, the policy maker has to rely on their experience, judgment, and the vast literature of political science. However, wise action is informed by good intelligence, and two tools deserve 
special mention in the context of managing complex adaptive systems: transparency, and computerassisted system modeling.

\section{Transparency}

System complexity means that a decision maker has limited insight into what's going on, and needs all the clues they can get; a forester must be able to read subtle signs of trouble, such as egg masses on trees in winter hinting of an infestation that would be invisible when the leaves are out in summer.

Transparency is known to be a useful tool for managing the volatility in complex systems. In the Beer Distribution Game, invented by Jay Forrester in the 1950s, volunteers play the roles of brewer, distributor, wholesaler, and retailer; each can adjust its orders up the chain at will to satisfy their perception of downstream demand (Sterman 2000: 684-708). ${ }^{79}$ At a certain point, the game manager doubles consumer demand; invariably, shipping volumes in the supply chain start oscillating wildly, and never stop. Sterman found that there are two ways to dampen these cycles: reduce the time delay between placing an order up the chain and receiving inventory; and give participants more visibility on what's happening in the supply chain.

Visibility into the workings of a complex system therefore reduces volatility and improves resilience. But in a rapidly-changing market system with lots of players, like the internet/web, how does one get timely information? Since funding for monitoring by regulators is limited, it is important to enable surveillance by civil society and the market itself. Limited capacity and visibility at the top of the control hierarchy can be complemented by distributing monitoring throughout the system.

It is particularly important to have effective disclosure mandates on powerful players, such as firms with significant market power and regulators themselves. Disclosure should be to the public domain. Disclosure limited to confidential regulator-regulated relationships is not helpful since it does not reach those who need it most: new entrants and users at the edge of the system.

Complex systems change simultaneously at a variety of speeds. It is important to monitor both fast and slow variables. Fast change, such as the loss of forest stand due to fire, is easy to monitor. Slower change, like the build-up over decades of an insect infestation that can lead to sudden, widearea defoliation, is harder to monitor but more influential. ${ }^{80}$ In the internet/web case, fast variables include market share, profitability, and company formation, merger and bankruptcy. An important slow variable is the emergence of market bottlenecks, eg, an applications or advertising platform that creates an indispensable multi-sided market.

Transparency is an increasingly fashionable policy tool. Research by the Harvard-based Transparency Policy Project (Fung et al. 2007) recommends that disclosure is only effective if responders are given accurate, actionable information. Disclosures should be understandable, and should match users' needs at the moment when they're making decisions. Disclosers should have the means and incentive to respond to users' choices; eventually, disclosers should come to see transparency as a business tool.

\footnotetext{
${ }^{79}$ For web resources, see eg http://www.pom.edu/beer/BeerDocs.htm and http://supplychain.mit.edu/games/beergame.aspx

${ }^{80}$ Ludwig, Jones and Holling (1978) showed that slow increases in spruce budworm infestation can lead to sudden forest collapse. When a forest is young and sparse, bird predation controls budworm numbers. As the forest matures, it becomes harder for birds to find the worms, which eventually leads to explosive growth in budworm numbers, and thus collapse of the forest. For more, see

http://www.geog.mcgill.ca/faculty/peterson/susfut/resilience/rBudworm.html and http://www.na.fs.fed.us/spfo/pubs/fidls/sbw/budworm.htm
} 
There are, of course, limitations. More transparency isn't necessarily better, and more information isn't synonymous with full disclosure. Transparency has a cost, both in terms of an additional burden on disclosers, and in unintended consequences. Many disclosure policies don't work; they're the result of political compromise, and powerful interests can neuter disclosure. It is also important to require sufficient disclosure without mandating the detailed format.

Internet/web technology itself facilitates monitoring. It makes information more immediately accessible, and enables peer-to-peer disclosure and the pooling of information and knowledge. The pioneers like Zagat, Amazon Reviews, and Wikipedia are being joined by "vigilante transparency" organizations monitoring civil servants, campaign contributions lobbyists, internet service providers and even nannies. ${ }^{81}$ Fung et al. (2007) argue that policy makers can and should make greater use of these approaches.

Transparency complements all the principles outlined above, but particularly Delegation and Flexibility. Since ideas stimulate more ideas, awareness of problems as well as opportunities will inspire invention and give participants to whom power has been delegated the opportunity to act wisely. Resilience increases when system participants can learn quickly about changes and failures, adapt, and benefit from them.

An abundant flow of information about the health of a system gives a regulator the information needed to adjust policy if necessary. Of course, policy flexibility does not presuppose regulatory intervention; a policy can be designed to flex on its own as the situation changes. However, visibility into both the situation and the policy's performance allows a regulator to learn, and adjust if necessary. For example, one can argue that regulatory intervention like supervising players with SMP should be limited to "enduring bottlenecks" - but since the system is in disequilibrium, it's very hard to predict whether a bottleneck exists, is coming about, or will endure. The best one can do is to observe carefully, and distribute the results quickly. In many cases the market will selfcorrect, but disclosure and distributed monitoring will speed up regulatory intervention should it become necessary.

Many transparency proposals are emerging in current internet/web policy debates. Atkinson \& Weiser's (2006) recommendations for a new approach to network neutrality include a transparency provision as part of effective consumer protection measures; they recommend that "all providers should state clearly to what extent content and services enjoy preferential delivery opportunities and any limitations on the ability of consumers to access the content and services of their choice." The FCC would then monitor whether firms comply in practice with their stated policies. Weiser (unpublished) recommends disclosure as part of a consumer protection strategy. Measures include guidance and supervision by the FTC regarding information consumers need, and accurate representations by broadband providers of the network management policies they use. PFIR launched the "Network Neutrality Squad"82 in November 2007 with the stated mission of the "detection, analysis, and incident reporting of any anticompetitive, discriminatory, or other restrictive actions on the part of Internet Service Providers (ISPs) or affiliated entities, such as the blocking or disruptive manipulation of applications, protocols, transmissions, or bandwidth; or other similar behaviors not specifically requested by their customers.” There is a pending petition for rulemaking from P2P video distributor Vuze Inc, which asks the FCC to examine network operators'

\footnotetext{
${ }^{81}$ Civil servants: http://nyc.uncivilservants.org. Campaign contributions: $\underline{\text { http://fundrace.huffingtonpost.com/. }}$ Lobbyists: http://www.opensecrets.org/lobbyists/. ISPs: http://www.nnsquad.org/. Nannies: http://isawyournanny.blogspot.com.

${ }^{82}$ http://www.nnsquad.org/
} 
network management practices, and make rules to prevent them from discriminating against particular internet applications, content, or technologies. ${ }^{83}$

In summary, transparency requires that consumers, civil society and the market be given access to information about the behavior of the policy/market system. This can be assisted by mandated disclosure of actionable information into the public domain, particularly by regulators, and firms with significant market power.

\section{Modeling}

All participants in a complex adaptive system have to innovate to stay ahead of ever-changing circumstances - policy makers too. However, every implemented solution has consequences. It leaves traces that cannot be undone. Once rules are in operation, the system will adjust to them, and there's no way to turn back the clock. Experimentation before deployment can reduce the risks of dramatic failure. Trying out new rules in limited geographies is a common approach. System modeling provides a new way to try out regulatory ideas safely, made possible by the advent of cheap, fast computing.

Simulations of the internet/web can help to improve policy makers' intuition of non-linear systems with many variables, including the slow ones that humans tend to miss. Exploring the consequences of policy choices in simulation can identify which courses of action are most robust under a variety of possible outcomes. Policy simulation allows decision makers to "sweat in training rather than bleed in combat." They can eliminate policy choices that are brittle and work in only a narrow set of circumstances, leading to more resilient final measures. Since any solution embodies a set of assumptions and biases, constructing a wide range of simulations can expose hidden preconceptions.

A variety of modeling techniques are available. The emerging discipline of systems dynamics (Sterman 2002) seeks to understand the behavior of complex systems through simulating the many interlocking, sometimes time-delayed, relationships among its components. It focuses on stocks and flows, with feedback loops among participants.

A number of social scientists have turned to agent-based modeling and simulation to examine social phenomena (Lempert 2002a, Sackler Colloquium 2002). Agent-based modeling simulates the interactions of many autonomous individuals on a network (Epstein \& Axtell 1996).

Bankes (2002) and Lempert (2002b) contend that robust policy strategies can only be developed by reasoning over an ensemble of policy scenarios. Inductive reasoning over large ensembles of computational experiments, known as Massive Scenario Generation (Davis et al. 2007), allows systematic comparison of alternative policy options. Davis et al. argue that this approach provides a way to address deep uncertainty, a situation where the system model and the input parameters to the system model are not known or widely agreed on by the stakeholders to the decision.

Simulation has so far hardly been used to explore the consequences of telecommunications policy decisions. Bauer 2007 explores the innovation incentives of network operators and content providers. He uses scenario thinking and simulation models to analyze the dynamics of various network neutrality policies. He offers a stylized model with subtle and nuanced qualitative discussion, but no simulation results are offered or described.

Shapira's 2004 MBA thesis uses MATLAB to integrate relatively simple simulations into a full blown system dynamics simulation of the telecom industry. He was able to reach conclusions about

\footnotetext{
${ }^{83}$ Filing 14 November 2007: http://fjallfoss.fcc.gov/prod/ecfs/retrieve.cgi?native or pdf=pdf\&id document=6519811711. eWeek story: http://www.eweek.com/article2/0,1895,2217747,00.asp.)
} 
the convexity of risk/return tradeoffs in R\&D investment under severe network externalities, as well as substitution as a matching device for innovation clock speed in competing industries. However, while he examines network externalities, investment incentives, standardization of products, and maximizing consumer welfare, he does not directly address a specific communications policy question like network neutrality.

There is an opportunity to apply the techniques developed for business modeling (Sterman 2002) and long-term policy analysis (Lempert et al. 2003) to the internet/web. For example, agent-based simulations of the internet access sub-system of the US telecoms industry could provide insight into the dynamics of network neutrality regulation. It would be instructive to explore whether the resulting system has multiple stable states, as one might expect from a complex adaptive system. There might be two (or more) stable consumer broadband industry structures at a given revenue level. One state might have a high degree of network neutrality and a low media concentration (ie, high diversity of content providers), while the other has more differential pricing and a higher media concentration (ie, lower diversity of content providers). As access provider revenue increases, content provider concentration increases in both states because the network operators are extracting more value from the system. There is a limit to the revenue possible for the high neutrality state, though, because most/all revenue is derived from consumers, and there is a limit to what they are willing to pay. Revenue and profit margins can only be increased by flipping to the low neutrality state, where access providers can extract additional revenue from content providers. This toy model is a riff on Scheffer's model of eutrophication of shallow lakes, which was discussed in Section V. C, Cycles and Transitions (Scheffer 1999). Once such a model is in place, one can extend it to do resilience analyses, and factor in political power (Scheffer et al. 2002).

\section{B. Parsing the Principles}

In the treatment of principles given so far, I have not distinguished between the different phases of policy making, or the different responsibilities of players in different roles.

\section{Phases in Policy Making}

One might broadly divide policy making into three phases: Policy development, which is the definition of social/political objectives (eg, increasing wealth by encouraging productive use of radio spectrum); legislation, specific statutes that seek to deliver on these goals (eg, legislation to authorize spectrum allocation through various means such as auctions); and regulation, which is the development and enforcement of detailed rules that implement legislation (eg, rules for auctioning spectrum and action to manage subsequent disputes). ${ }^{84}$

In the following table, the principles developed in Section VI above, The Four Principles, are teased apart into the actions most applicable to each phase. As the focus shifts from policy development to regulation, the guidance becomes more granular.

\footnotetext{
84 The boundaries are not hard and fast. In particular, the distinction between legislation and regulation is slippery in practice.
} 
Table 4: Principles partitioned into actions appropriate for different phases of policy making

\begin{tabular}{|c|c|c|c|}
\hline & Policy Development & Legislation & Regulation \\
\hline Flexibility & $\begin{array}{l}\text { Determine ends, not means. } \\
\text { Describe and justify the } \\
\text { outcomes sought, not the } \\
\text { methods to be used to } \\
\text { achieve them. }\end{array}$ & $\begin{array}{l}\text { Define expectations clearly, } \\
\text { but don't specify } \\
\text { implementation details. } \\
\text { Prefer ex post to ex ante. } \\
\text { Build in sunset dates and } \\
\text { glide paths. }\end{array}$ & $\begin{array}{l}\text { Use principles rather than } \\
\text { rules. Ensure backstop } \\
\text { powers are available if self- } \\
\text { regulation fails. Rules, if } \\
\text { used, should be technology } \\
\text { and business-model neutral. } \\
\text { Build in capacity to deal with } \\
\text { the unexpected. }\end{array}$ \\
\hline Delegation & $\begin{array}{l}\text { Presume that most problems } \\
\text { will be solved by the market } \\
\text { and society, not by } \\
\text { government. Identify } \\
\text { remaining distortions and } \\
\text { failures. }\end{array}$ & $\begin{array}{l}\text { Empower market and society } \\
\text { to solve problems. Provide } \\
\text { backstop powers to } \\
\text { regulators to address } \\
\text { distortions and failures. }\end{array}$ & $\begin{array}{l}\text { Intervene if players close to } \\
\text { the action fail to solve } \\
\text { problems. }\end{array}$ \\
\hline Big Picture & $\begin{array}{l}\text { Take a broad view of } \\
\text { problem/solution space. } \\
\text { Recognize different time and } \\
\text { commercial scales. }\end{array}$ & $\begin{array}{l}\text { Prefer generic to sector-, } \\
\text { technology-, or business- } \\
\text { specific legislation. }\end{array}$ & Avoid silo-specific regulation. \\
\hline Diversity & $\begin{array}{l}\text { Recognize that multiple } \\
\text { solutions are possible. } \\
\text { Encourage competition and } \\
\text { market entry. }\end{array}$ & $\begin{array}{l}\text { Allow and encourage } \\
\text { multiple solutions. Establish } \\
\text { principles for addressing } \\
\text { market failure. }\end{array}$ & $\begin{array}{l}\text { Don't entrench one solution } \\
\text { through regulatory } \\
\text { preference. Define markets } \\
\text { broadly for competitive } \\
\text { analysis. }\end{array}$ \\
\hline
\end{tabular}

\section{Roles in Policy Making}

Participants in different roles have different needs: a government minister, like any executive, needs to set strategy and evaluate performance, whereas a member of staff needs to devise rules that execute on the strategy. The principles are intended to be helpful in both cases. They can be used both to construct strategies and rules, and to evaluate their efficacy. For example, the recommendation of the Flexibility principle that rules be technology and business-model neutral can be used both to develop regulation, and test whether the resulting rules are, in fact, neutral.

The following list of questions can be used to evaluate policy, legislative or regulatory proposals to ensure that they conform to the principles:

Table 5: Questions to check that policy proposals conform to the principles

\begin{tabular}{llll}
\hline & Policy Development & Legislation & Regulation \\
\hline Flexibility & Are the desired outcomes & Are the desired outcomes & Are principles favored over \\
& clearly stated? Are & clearly stated? Are & rules? Are rules that are used \\
implementation & prescriptive solutions & technology and business- \\
& prescriptions avoided? & avoided? Is ex post action & model neutral? Can \\
& favored, and authorized? Are & regulation adapt to deal with \\
& sunset dates prescribed, and & the unexpected? \\
& glide paths allowed? &
\end{tabular}


Delegation Is the presumption that most problems can be solved by

Big Picture

Diversity

Does the outcome statement take a broad view of problem/solution space? Does it recognize the diverse ways in which the system operates? the market and society?

Is power to solve problems delegated to market and society? Does government retain the back-up power to correct distortions and failures?
Is legislation generic rather than sector-, technology-, or industry-specific?
Does legislation recognize that multiple solutions are possible? Does it encourage competition and market entry?
Are individuals empowered to solve problems in the first instance? Does the regulator have the power to Intervene if players close to the action fail to solve problems?

Is regulation (incl. revenue generation) generic rather than sector-, technology-, or industry-specific?

Does regulation avoid favoring one solution through regulatory preference? Does it define markets broadly?

\section{C. Using the Principles outside Government}

This paper aims to show that complex systems thinking, made concrete through metaphors like forestry, can provide policy makers with a practical framework for watching over the internet/web. However, the principles are a useful guide for all internet/web players, including companies and consumer advocates.

In complex adaptive systems, all agents operate from behind a veil of ignorance about the details of the situation they find themselves in, and the outcome of their actions. ${ }^{85}$ One cannot predict outcomes with any certainty in a complex system; they are sensitive to initial conditions, agents in the system constantly push back against changes, and surprise is inevitable. While one might be able to predict small, near-term variations, the high-impact large changes are not predictable.

Two conclusions follow: first, that any participant needs some unchanging principles that will tend to give good results regardless of the constantly changing circumstances; and second, that calculations based on perceived self-interest may turn out to be wildly wrong, since the agent's longterm interests may be at variance with short-term benefits.

Companies frequently have "Oh, never mind" moments that reflect changes of mind triggered by changes in their situation. ${ }^{86}$ For example, in the late 90 s, AOL lobbied to require cable networks to give ISPs the right to offer services via cable networks. After TW and AOL announced their merger in 2000, Case retreated and the AOL lobbyists had to eat their words. ${ }^{87}$ Another recent example is

\footnotetext{
${ }^{85}$ The veil of ignorance was introduced by Rawls (1971/1999). Its applicability here does not depend on Rawls's approach or conclusions. His veil was a thought experiment; in complex adaptive systems, the veil of ignorance is a fact of life.

${ }^{86}$ Comic actress Gilda Radner's character Roseanne Roseannadanna.on Saturday Night Live would deliver agitated editorial commentary on some topic, only to discover half-way through that she had misheard the issue. When the error was pointed out to her, she would say, “Oh, never mind.” See eg http://en.wikipedia.org/wiki/Gilda_Radner. The usage in this context is due to Marc Berejka.

${ }^{87}$ Perine, Keith (2000) “You’ve got scrutiny” The Industry Standard, March 13, 2000. Available: http://theindustrystandard.com/article/0,1902,12594,00.html. "Before the merger was announced in January, Case was the self-described Paul Revere of mandated open access, championing federal legislation before congressional committees to force cable companies to let ISPs use their broadband networks. AOL actively lobbied for such rules,
} 
the cellular industry's about-face on local number portability (LNP). Mobile phone companies had argued strenuously before the FCC that requiring them to allow customers to keep their numbers when they changed carriers was technically impossible, and would be ruinously expensive to implement even if it were possible. The real reason, of course, was that everyone feared that LNP would increase churn and reduce profit. However, once Verizon broke ranks - it had led the protracted, industry-wide effort to prevent the FCC from requiring LNP - everybody else in the industry quickly came around. ${ }^{88}$ In the face of the uncertainty inherent in complex adaptive systems, then, reliance on principles is not only good strategy but may also minimize embarrassing flip-flops.

Short-term calculations of self-interest remain vital to tactical competition. However, players can build a productive and resilient position defining and adhering to strategies based on balanced principles like those described here. This is true not only for companies: it holds for anyone taking a strategic view of the interests of their constituency, from governments and politicians to consumer advocacy groups and NGOs.

\section{D. Beyond the Internet/web}

Section III. A, Characteristics of the Internet/Web, argued that the following changes in communications necessitated a new regulatory framework:

- Modularity. The combination of prolific innovation (often driven by software) and standard interfaces allows new kinds of functionality to be composed rather than having to be built in an integrated way from the ground up (YouTube vs. cable TV). User experiences are built like nesting dolls, with services depending on sub-services, sub-sub-services etc. that they do not control. This modularity correlates with, and encourages, more numerous and diverse market participants.

- Self-organization. Industry structure emerges at the edges, as firms focused on modules spring up and undermine business models built on vertical integration (Microsoft vs. IBM, Google vs. Madison Avenue, YouTube vs. Hollywood). Citizens are no longer just consumers; they're also producers (user-generated content like Amazon recommendations, digg, and MySpace pages).

- Rapid change. New technology-business architectures and decentralized organization are speeding up the rate of change, surging ever further ahead of the pace of regulation. While the system is probably in a hyper-fast phase at the moment, it will never relax back to the pace of the 1950s.

These changes are being driven by software-based services, ever-more powerful digital machines, standardized data streams on global broadband networks, and a flourishing world of intangibles. These technologies are replacing an economy built out of analog hardware, static content, slow connections, and national or regional business geography. We are transitioning from a system that is largely concrete, deterministic, slow moving, and linear to one that is largely intangible, unpredictable, volatile, and non-linear: from an industrial economy to a knowledge economy.

retreating only after it decided to buy the opposition. Last week, Case was forced to backpedal. He told both Senate committees that he always preferred voluntary open access by the cable industry, and only if that didn't occur would a "light touch" of federal law be needed. Case pointed to the new memo as proof that the industry - namely Time Warner and its chief cable rival, ATT - is committed to open access. ... Several senators on both committees were openly skeptical of the open-access pledge, which is not legally binding.”

${ }^{88}$ See eg “In a Reversal, Verizon Backs Rule to Keep Cell Numbers”, Matt Richtel, New York Times, 25 June 2003. Available at http:/query.nytimes.com/gst/fullpage.html?res=9402EED9133BF936A15755C0A9659C8B63 
It follows that some or all regulation will have to be rethought in any industry where such phenomena are occurring, particularly those where software, silicon and connectivity are driving reorganization. This has already happened in financial services, and is beginning to happen in health care, retail, and energy.

The old value chains were relatively easy to regulate because they only had a small number of parts that moved in concert, if they moved at all. Fragmentation vastly increases the number of entities, which in turn exponentially increases the number of links and sockets. Links and sockets are the points of leverage for regulation. This creates the regulatory challenge that the Ecosystem Management Principles are intended to address. One cannot regulate the detailed behavior or interactions of the numerous, ever-changing entities in an adaptive complex system; the only viable solution is to move up a level and think in terms of desired outcomes - that is, principles rather than rules.

The Ecosystem Management Principles were developed to balance competing needs in the arena where such change is occurring most visibly: the internet/web. However, they apply to any industry that is experiencing the breakdown of previously predictable, synchronous value chains into highly fragmented, unpredictable, asynchronous communities.

\section{OPEN QUESTIONS}

The approach outlined here raises many questions that have not been fully addressed. This section outlines some opportunities for future work.

\section{A. Testing the Metaphor}

This paper illustrates the applicability of its method by examining a few practical cases at a high level. A detailed analysis of a particular case, which would include a comparison of old regulation, current regulation, and new regulations that these principles would suggest, is required to prove the worth of this approach. The predictions and prescriptions of this approach need to be compared and contrasted with alternative policy frameworks, such as the architecture-as-policy (layers) and freemarket approaches.

The policy recommendations lean towards a laissez faire "Anglo-American" approach: skepticism about the ability of government to intervene effectively through central planning, a bias towards light-handed regulation, and empowering the market and individuals to solve problems. Is this a necessary consequence of a systems approach, or could the same premises in different hands have led to dirigiste "European" prescriptions: a belief in the need for government to manage markets, a willingness to regulate, and a social democratic rather than capitalist world view?

What policy solutions are ruled out by this approach? If any set of principles and policy tools can be derived equally plausibly from the premise that the internet/web is managed complex adaptive system, then the approach has little descriptive, predictive or normative value.

\section{B. Guiding Policy Making}

One of the requirements of flexible policy is that it should work equally well in times of growth, maturity, collapse, and renewal. Can the management of macro-economic cycles in the financial sector inform the regulation of communications?

This paper proposes ways to deal with policy problems, but takes the problems themselves as a given. However, policy makers need to diagnose and characterize challenges before they can address them. Can complex systems thinking help policy makers delineate the problem? 
It needs to be shown that the Ecosystem Management Principles can help policy makers deliver on the public interest imperatives (Section II, Permanent Policy Imperatives) in general. Their utility in specific problems is encouraging, but this does not demonstrate that they are a general-purpose solution to meeting public interest mandates.

\section{C. Applying System Theory to the Internet/Web}

There is a great deal of scope for the ecosystem management metaphor to generate new research questions, indicate policy solutions new to the internet/web discourse, and make falsifiable predictions.

If the internet/web is a complex adaptive social system that resembles a managed ecosystem, then many of the techniques developed in that field should be transferable. What are suitable metrics for tracking the overall (emergent) performance of the internet/web? Are there metrics for overall "quality" of the internet/web that relate to emergent properties? Can one simplify the complexity of the internet/web by identifying the key processes, and a limited number of variables that control them, at a variety of scales in a hierarchical structure?

Panarchy theory suggests that variables operating at different speeds are important to adaptive cycles; for example, the key variables in forest-pest dynamics are insects (fastest), foliage (slower), and trees (slowest). ${ }^{89}$ Does the internet/web have similar dynamics? Can one diagnose which part of the cycle various sub-systems find themselves in? What policy recommendations follow?

Complex system theory suggests that the internet/web industry should contain bi-stable states. The paper suggests that this was the case for video production and distribution, but the claim needs to be validated by a detailed econometric analysis. Are there other cases? It is also necessary to analyze boom/bust cycles in the ICT industry in the light of adaptive cycles. Candidates include the music publishing industry ${ }^{90}$, telephone company revenue streams ${ }^{91}$, and theatrical entertainment ${ }^{92}$.

Any systems analysis requires one to draw boundaries around sub-systems. On the other hand, the flux of the current internet/web makes classifications protean, and borders fuzzy. ${ }^{93}$ Clark et al. (2005), for example, recommend that internet system design should be modularized along "tussle boundaries". How does this relate to a layers analysis? How does it interact with the classificatory instinct of regulators? One of the difficulties of the "European approach" to communications regulation, and with antitrust rules in general, is the definition of a market. Any definition necessarily draws an artificial boundary around a system in constant flux, a particularly difficult task during periods of rapid innovation. Can complex system theory cast light on ways to define markets that take dynamics into account?

System dynamics techniques that work well to model situations with relatively stable constituents and clear boundary conditions (such as established industries and geopolitics) may fail

\footnotetext{
${ }^{89}$ See eg Gunderson \& Holling (2001:69) Table 3-1

${ }^{90}$ The labels are seeing a collapse in CD sales. Prior booms and busts in the music industry may include the introduction of sheet music undermining the power of performers while helping composers and publishers, and the introduction of recordings putting a dent in concert hall revenues.

${ }^{91}$ Telcos have seen (and will continue to see) their voice revenue collapse, and data revenues have not kicked in yet. A similar transition happened from telegraphy to voice traffic.

${ }^{92}$ Waves of technology have swept through "theatrical” entertainment, including film, radio, television, and the internet.

${ }^{93}$ Frieden (2007) contends that the old dichotomy between regulated "telecom services” and unregulated "information services" is no longer sustainable. Technological convergence and innovations calls into question whether Congress can create categories that the FCC can use to determine the scope of government oversight, and the FCC itself has exacerbated the confusion with some of its rulings.
} 
when applied to the internet/web, which is heterogeneous and evolving rapidly. Even if the models can be constructed, they may become obsolete rapidly. All systems are unstable and porous, though; only experience will show whether is internet/web (or some chosen part of it) is too dynamic to be tractable.

\section{D. Cross-border Governance}

Many ecosystem management problems cross borders, and much can be learned from this experience to help international governance issues on the internet/web. This fascinating topic will not be addressed in any detail in this paper; a brief discussion will have to suffice.

The essence of the problem is that sovereigns have local purview, but need to optimize globally to solve problems. As one learns from system theory, a system as a whole will not operate a maximum efficiency if each sub-system is locally optimized (Skyttner 2001:92). Natural systems do rather well by optimizing locally, and one might expect that this would be acceptable for nations, too. However, national sovereigns have reasons beyond efficiency for their actions, such as history, cultural preferences, and political self-interest; these complicate the situation and may lead to a suboptimal global solution. Further, since the borders between states (physical and virtual) are porous, national isolation is impossible even if it were desirable. There are often contending philosophies that advance competing global-scale solutions, such as differing approaches to intellectual property, anti-trust, and privacy in the US and the EU.

The Ecosystem Management Principles suggest the following strategies

- Governments should delegate technical standards to international expert bodies: since plumbing isn't a governmental expertise, delegation would yield more efficient results; and since scale of plumbing is global, and it cannot be locally optimized. However while standards bodies might define how data moves and how content can be controlled, sovereigns should retain control over which data moves and which kinds of content are controlled.

- The Flexibility principle would suggest that desired outcomes be clearly stated, and powers reserved for action after the fact should it be necessary, rather than regulating in anticipation of unwanted developments.

- $\quad$ The Big Picture principle suggests that governments take a broad view of problems, and particularly of solutions, and avoid ad hoc regulation. The gains from global trade are an example of holistic benefits - and the problems of Doha trade round are a lesson in the difficulty of implementing such insight in the face of domestic politics.

- $\quad$ Some degree of heterogeneity between countries is desirable in order to maintain overall robustness via diversity; economic efficiency arguments for global harmonization - even if they trumped local political interests - have to be balanced by the need for system resilience. 


\section{CONCLUSIONS}

Policy makers frame questions and responses in terms of mental models. The complexity of the internet/web, particularly compared to traditional communication systems, requires new ways of thinking. The silo model shaped much of 20th century communications policy, and the layers model has been widely discussed in recent years. However, neither these models nor prevailing metaphors for the internet as highways, places or conversations fully capture the dynamic, human-managed complexity of the internet/web.

Both the internet/web and forests are managed complex adaptive systems. Ecosystem management theory has had to reconcile complex biological dynamics with relentless political intervention, and is thus a fruitful source of metaphors and lessons for internet/web policy. The silos of traditional communication regulation are like commercial farming: there are a limited number of well-defined fields and crops, ownership and success metrics are clear, and unintended consequences are rare. On the other hand, today's internet/web is like a global patchwork of national parks and forests. The regulator is more forester than farmer. Control is decentralized, there are many competing resource uses, and the results of an intervention are uncertain.

The findings of systems theory and ecosystem management inspire four principles that can help internet/web policy makers balance competing demands: flexibility (determine ends not means), delegation (give markets and society the first shot at solving problems), big picture (take a holistic view of the problem), and diversity (encourage competition and market entry). The principles can be used both to develop and evaluate policy; and they can be segmented into the actions most appropriate for different phases of the policy process.

These principles yield actionable recommendations when applied to current policy questions such as video services, fees on communications, accessibility for the disabled, and access to emergency services. The paradigm suggests forbearing from imposing legacy mandates such as taxes and accessibility requirements on new services, but only as long as they are nascent - just as a forester might protect seedlings, but let young trees fend for themselves. Just as occasional fires renew species diversity and promote long-term forest health, it recognizes that the disruption of favoring new entrants over incumbents can promote overall system resilience. Humility in the face of the constant novelty of complex systems militates against before-the-fact rules, and argues for termination dates on regulations.

The Ecosystem Management Principles are necessary but not sufficient; they need to be combined with long-standing techniques of good governance such as transparency, consistency, accountability and proportionality. They should be supported by computer modeling, which provides a deeper understanding of the complex dynamics of contemporary regulatory challenges.

The principles have been laid out here in the context of government policy making for the internet/web. However, they apply in any situation where technology drives rapid change in a complex managed system, such as decision making in enterprises, and the regulation of any industry that is being transformed by information technology.

\section{ACKNOWLEDGMENTS}

This paper is built with the ideas of the authors listed in the bibliography and footnotes; I'm indebted to them all. I'm grateful to the many people who have contributed support, comments and insights to this work, including an anonymous reviewer, Jonathan Aronson, Brad Bernthal, Paula Boyd, Chee Chew, Matthew Clark, Stephen Coller, Peter Cowhey, Theo Eicher, Tren Griffin, Jonathan Grudin, Anoop Gupta, Scott Blake Harris, Peter Haynes, Pam Heath, Scott Marcus, Paul 
Margie, Jim Miller, Adam Thierer, Ed Thomas, Susan Tonkin, Phil Weiser, and Simon Wilkie. If their helpful suggestions have not been taken up, it is due to my inadequacies and not the quality of their advice. In addition to their contributions to the paper, I'm particularly grateful to Kevin Minsky for providing the impetus for this work, and to Marc Berejka who's been telling me for years that complex system theory is important for policy. The work was funded in part by a grant from Microsoft Corporation to the Economic Policy Research Center at the University of Washington.

\section{REFERENCES}

Amendola, Giovanni Battista \& Lorenzo Maria Pupillo (2007) “The Economics of Next Generation Access Networks and Regulatory Governance in Europe: One Size Does not Fit All” 35th Research Conference on Communication, Information and Internet Policy Washington DC 28-30 September 2007 (TPRC 2007). Available: http://web.si.umich.edu/tprc/papers/2007/804/Amendola_Pupillo_\%2035TPRC.pdf

Andersen J.V. \& D. Sornette (2005) "A mechanism for pockets of predictability in complex adaptive systems” Europhys. Lett. vol. 70 pp. 697-703

Anderson, Tom, Scolt Shenker, Ion Stoica and David Wetherall (2003) "Papers from Hotnets-1 - Design Guidelines for Robust Internet Protocols” Computer communication review vol. 33, no. 1, p. 125 (2003)

Arthur, W.B. (1994) Increasing Returns and Path Dependence in the Economy, University of Michigan Press, Ann Arbor.

Atkinson, Robert D. \& Philip J. Weiser (2006) “A ‘Third Way’ on Network Neutrality” 30 May 2006. Available: http://www.itif.org/files/netneutrality.pdf

Bankes, Steven C. (2002) "Tools and techniques for developing policies for complex and uncertain systems” Proc Natl Acad Sci U S A, May 14, 2002, vol. 99, suppl. 3, 7263-7266

Bauer, Johannes M. (2007) “Dynamic effects of network neutrality” International Journal of Communication, vol. 1, pp. 531-547. Available: http://ijoc.org/ojs/index.php/ijoc/article/view/156/79. Summary presentation at http://www.msu.edu/ bauerj/presentations/bauer-tprc-2006-presentation.pdf

Beinhocker, E. D. (2006) The Origin of Wealth, Harvard Business School Press

Brock, W.A. (2001) “The Telephone Great Fits the Figure Eight” sidebar in Holling et al. (2001)

Carlson J.M. \& J. Doyle (2002) “Complexity and Robustness” Proc Natl Acad Sci, 99, suppl. 1, pp. 25382545. Available: http://www.cds.caltech.edu/ doyle/CmplxNets/pq04u2002538.pdf.

Clark, D.D., J. Wroclawski, K.R. Sollins, and R. Braden (2005) "Tussle in cyberspace: defining tomorrow's Internet” IEEE/ACM Transactions on Networking, vol. 13, no. 3, pp. 462-475, Jun. 2005. Available: http://www.cs.princeton.edu/courses/archive/spr06/cos592/bib/tussle-p347clark.pdf

Cowhey, Peter F., Jonathan D. Aronson and John Richards (manuscript) The Inflection Point: Global Governance in a Digital Age

Crawford, Susan P. (2007) "Internet Think" Journal on Telecommunications and High Technology Law, 2007. Available: http://ssrn.com/abstract=962596

Cukier, Kenneth Neil (2007) "Governance as Gardening: A Report of the 2007 Rueschlikon Conference on Information Policy” The Rueschlikon Conferences on Information Policy in the New Economy. Available: http://www.cukier.com/writings/Rueschlikon2007-infogov-cukier.pdf

Davis, Paul K., Steven C. Bankes, and Michael Egner (2007) "Enhancing Strategic Planning with Massive Scenario Generation: Theory and Experiments” RAND Technical Report TR-392, 2007. Available: http://www.rand.org/pubs/technical_reports/TR392/

De Vries, P. (2006) "Populating the Vacant Channels: The Case for Allocating Unused Spectrum In the Digital TV Bands to Unlicensed Use for Broadband and Wireless Innovation” New America 
Foundation Working Paper, Aug. 8, 2006. Available:

http://www.newamerica.net/publications/policy/populating_the_vacant_channels

Doyle J. \& M. Csete (2007) "Rules of engagement” Nature vol. 446, p. 860, 19 Apr. 2007

Economides, Nicholas (1998) "The Telecommunications Act of 1996 and its Impact" (September 1998). New York University, Center for Law and Business, Working Paper no. 99-003. Available: http://ssrn.com/abstract $=81289$

Epstein, J., \& Axtell, R. L. (1996) Growing artificial societies: Social science from the bottom up, MIT Press

European Commission (1997) "Green paper on the convergence of the telecommunications, media and technology sectors, and implications for regulation: Towards an Information Society Approach” COM vol. 97, p.623, Brussels, 3 Dec. 1997. Available: http://rspg.groups.eu.int/doc/documents/legal/conver97.pdf

European Parliament (2002) "Directive 2002/21/EC of the European Parliament and of the Council of 7 March 2002 on a common regulatory framework for electronic communications networks and services (Framework Directive)" Official Journal L 108 , 24/04/2002, p. 33-50. Available: http://eur-lex.europa.eu/LexUriServ/LexUriServ.do?uri=CELEX:32002L0021:EN:NOT

Faratin, Peyman, David Clark, Patrick Gilmore, Steve Bauer, A. Berger and William Lehr (2007) "Complexity of Internet Interconnections: Technology, Incentives and Implications for Policy" 35th Research Conference on Communication, Information and Internet Policy Washington DC 28-30 September 2007 (TPRC 2007). Available: http://web.si.umich.edu/tprc/archive-searchabstract.cfm?PaperID=797

Farrell, Joseph \& Phil Weiser (2003) “Modularity, Vertical Integration, and Open Access Policies: Towards a Convergence of Antitrust and Regulation in the Internet Age" Harvard Journal of Law and Technology, vol. 17, no. 1, Fall 2003 Available: http://ssrn.com/abstract=452220

Frieden, Rob (2007) "Neither Fish nor Fowl: New Strategies for Selective Regulation of Information Services" 35th Research Conference on Communication, Information and Internet Policy Washington DC 28-30 September 2007 (TPRC 2007). Available: http://web.si.umich.edu/tprc/papers/2007/670/Neither\%20Fish\%20Nor\%20Fowl.pdf

Fung, Archon, Mary Graham and David Weil (2007) Full Disclosure: The Perils and Promise of Transparency, Cambridge University Press

Gunderson, Lance \& C.S. Holling (eds.) (2001) Panarchy: Understanding Transformations in Human and Natural Systems, Island Press

Hazlett, Thomas W. (2000) "Economic and Political Consequences of the 1996 Telecommunications Act” Regulation, vol. 23, no. 3, 2000. Available: http://papers.ssrn.com/sol3/papers.cfm?abstract_id=244818

Hazlett, Thomas W (2008) “Optimal Abolition of FCC Spectrum Allocation”, Journal of Economic Perspectives, vol. 22, no. 1, pp. 103-128, Winter 2008

Hesse, Mary (1966) Models and Analogies in Science. Notre Dame University Press

Holling C.S., Gunderson L.H., and Ludwig D. (2001) "In Quest of a Theory of Adaptive Change” Chapter 1 in Gunderson \& Holling (2001). Available: http://www.resalliance.org/593.php

Holling C.S. and L.H. Gunderson (2001) "Resilience and Adaptive Cycles” Chapter 2 in Gunderson \& Holling (2001).

Homer-Dixon, Thomas F. (2006) The upside of down: catastrophe, creativity, and the renewal of civilization, Island Press.

Iansiti, Marco and Roy Levien (2004) The Keystone Advantage: What the New Dynamics of Business Ecosystems Mean for Strategy, Innovation, and Sustainability, Harvard Business School Press, 2004. Chapter 1 available:

http://harvardbusinessonline.hbsp.harvard.edu/b01/en/common/viewFileNavBean.jhtml;requestid=2 $\underline{26972}$ 
Jain, S. and S. Krishna (2002) "Crashes, recoveries, and "core shifts" in a model of evolving networks" Physical Review E vol. 65, no. 2, 026103, 9 Jan 2002, cited in Beinhocker (2006)

Klamer A. \& T. C. Leonard (1994) “So what's an economic metaphor?” in Natural Images in Economic Thought, P. Mirowski (ed.) Cambridge University Press

Lakoff G. and M. Johnson (1980) Metaphors We Live By, University Of Chicago Press; 2nd edition

Lempert, Robert J. (2002a) “Agent-based modeling as organizational and public policy simulators” Proc Natl Acad Sci U S A, May 14, 2002; vol. 99 (Suppl. 3) pp. 7195-7196

Lempert, Robert J. (2002b) “A new decision sciences for complex systems” Proc Natl Acad Sci U S A, May 14, 2002; vol. 99 (Suppl. 3) pp. 7309-7313

Lempert, Robert J., Steven W. Popper, and Steven C. Bankes (2002) “Confronting Surprise” Social Science Computer Review, no. 4, 2002, pp. 420-440

Lempert, Robert J., Steven W. Popper, and Steven C. Bankes (2003) "Shaping the Next One Hundred Years: New Methods for Quantitative, Long-Term Policy Analysis” RAND Report MR-1626-RPC, 2003. Available: http://www.rand.org/pubs/monograph_reports/MR1626/index.html.

Ludwig, D., D.D. Jones and C.S. Holling (1978) “Qualitative analysis of insect outbreak systems: the spruce budworm and forest” Journal of Animal Ecology 47(1):315-332.

Marcus, J. Scott (2002) "The Potential Relevance to the United States of the European Union's Newly Adopted Regulatory Framework for Telecommunications,” OPP Working Paper Series no. 36, Jul. 2002

Marcus, J. Scott (2006) "Beyond Layers" (9 May, 2006). Available: http://ssrn.com/abstract=901477

Mirowski, P. (1989) More Heat than Light. Cambridge University Press

Ness, Susan (2006) “The Law of Unintended Consequences” Federal Communications Law Journal vol. 58, no. 3, p. 531, June 2006. Available at: http://www.law.indiana.edu/fclj/pubs/v58/no3/k-ness.pdf

Nicholas, Tom (2003) "Why Schumpeter was Right: Innovation Market Power and Creative Destruction in 1920s America” The Journal of Economic History, vol. 63, no. 04, pp 1023-1058, Dec 2003

Odlyzko, Andrew (n/d) "Layer architectures and regulation in telecommunications" not dated. Available: http://www.dtc.umn.edu/ odlyzko/doc/layer.architecture.txt

Perez, C. (2002) Technological Revolutions and Financial Capital: The Dynamics of Bubbles and Golden Ages, Edward Elgar

Pollan, Michael (1991) Second Nature: A Gardener's Education, Atlantic Monthly Press

Rawls, John (1971/1999) A Theory of Justice (Revised Edition) Belknap Press

Richardson, G. (1991) Feedback Thought in Social Science and Systems Theory, University of Pennsylvania Press; also Pegasus Communications (1999, paperback)

Rittel, Horst W.J. and Melvin M. Webber (1973) “Dilemmas in a General Theory of Planning” Policy Sciences vol. 4 (1973), pp. 155-169.

Sackler Colloquium of the National Academy of Sciences (2002) "Adaptive Agents, Intelligence, and Emergent Human Organization: Capturing Complexity through Agent-Based Modeling” Proc Natl Acad Sci U S A May 14, 2002; vol. 99 (Suppl. 3) pp. 7178 ff.

Scheffer, M. (1999) "Searching explanations of nature in the mirror world of math" Conservation Ecology, vol. 3, no. 2, p. 11. Available: http://www.consecol.org/vol3/iss2/art11/.

Scheffer, M., F. Westley, W. A. Brock, and M. Holmgren (2001) "Dynamic Interaction of Societies and Ecosystems - Linking Theories from Ecology, Economy, and Sociology" Chapter 8 in Gunderson \& Holling (2001).

Schlag P. (2002) "The Aesthetics of American Law” Harv. L. Rev. vol. 115, p. 1047

Shapira, Gil (2004) "System Dynamics Simulation of the Telecom Industry" MS thesis, submitted to the MIT Sloan School of Management, 7 May 2004. Available:

http://itc.mit.edu/itel/students/papers/Shapira thesis.pdf 
Skyttner, L. (1996). “General systems theory: origin and hallmarks” Kybernetes. vol. 25, no. 66, pp. 1622

Skyttner, L (2001). General Systems Theory: Ideas \& Applications, World Scientific Publishing

Snider, J H (2007) “The Art of Spectrum Lobbying: America's \$480 Billion Spectrum Giveaway, How it Happened, and How to Prevent it from Recurring,” New America Foundation, Aug. 2007. Available: http://www.newamerica.net/publications/policy/art_spectrum_lobbying

Solum Lawrence B. \& Minn Chung (2003) “The Layers Principle: Internet Architecture and the Law” University of San Diego School of Law, Public Law and Legal Theory Research Paper no. 55, Jun. 2003. Available: http://ssrn.com/abstract $=416263$.

Sterman, J.D. (2000) Business Dynamics, McGraw Hill

John D. Sterman (2002) “All models are wrong: reflections on becoming a systems scientist: The Jay Wright Forrester Prize Lecture.” System Dynamics Review vol. 18, no. 4, pp. 501-531 (Winter 2002)

Taleb, Nassim Nicholas (2001) Fooled by Randomness: The Hidden Role of Chance in Life and in the Markets, WW Norton \& Co

Tether, B. S. (1998) “Small and large firms: sources of unequal innovations?” Research Policy, vol. 27, no. 7, pp. 725-745, November 1998

Walker, Brian and Nick Abel (2001) “Resilient Rangelands - Adaptation in Complex Systems” Chapter 3 in Gunderson \& Holling (2001)

Weiser, Philip J. (unpublished) “The Next Frontier for Network Neutrality”

Wells, Peter E. and Lauren Darby (2006) "Re-writing the ecological metaphor, Part 2: the example of diversity” Progress in Industrial Ecology - An International Journal, vol. 3, nos. 1/2, p. 129, 2006

Werbach, Kevin D. (2002) “A layered model for Internet policy” Journal on Telecommunications and High-Tech Law, vol. 1, no. 37, 2002, Available: http://ssrn.com/abstract=648581. A draft of this article was presented in September 2000 at the 28th Annual Research Conference on Information, Communications, and Internet Policy (TPRC 2000), available: http://www.tprc.org/abstracts00/layeredpap.pdf.

Whitt, Richard S. (2004) “A Horizontal Leap Forward: Formulating a New Communications Public Policy Framework Based on the Network Layers Model” Federal Communications Law Journal, vol. 56, no. 3, p. 587, May 2004. Available: http://law.indiana.edu/fclj/pubs/v56/no3/Whitt\%20Final\%202.pdf

Whitman J Q (2004) “The two Western cultures of privacy: Dignity versus liberty” Yale Law Journal vol. 6, p. 1151, Apr. 2004

Winter, S. L. (2003) A Clearing in the Forest, University of Chicago Press

Guo, Zhenzhi (2003) "Playing the game by the rules? Television regulation around China's entry into WTO” Journal of the European Institute for Communication and Culture, Javnost - The Public vol. 10 (2003) no. 4, p. 5-18. Available: http://www.javnost-thepublic.org/media/datoteke/guo-42003.pdf 